\title{
Total domination and matching numbers in claw-free graphs
}

\author{
${ }^{1}$ Michael A. Henning* and ${ }^{2}$ Anders Yeo \\ ${ }^{1}$ School of Mathematical Sciences \\ University of KwaZulu-Natal \\ Pietermaritzburg, 3209 South Africa \\ henning@ukzn.ac.za \\ ${ }^{2}$ Department of Computer Science \\ Royal Holloway, University of London, Egham \\ Surrey TW20 OEX, UK \\ anders@cs.rhul.ac.uk
}

Submitted: Apr 18, 2006; Accepted: Jun 30, 2006; Published: Jul 28, 2006

Mathematics Subject Classification: 05C69

\begin{abstract}
A set $M$ of edges of a graph $G$ is a matching if no two edges in $M$ are incident to the same vertex. The matching number of $G$ is the maximum cardinality of a matching of $G$. A set $S$ of vertices in $G$ is a total dominating set of $G$ if every vertex of $G$ is adjacent to some vertex in $S$. The minimum cardinality of a total dominating set of $G$ is the total domination number of $G$. If $G$ does not contain $K_{1,3}$ as an induced subgraph, then $G$ is said to be claw-free. We observe that the total domination number of every claw-free graph with minimum degree at least three is bounded above by its matching number. In this paper, we use transversals in hypergraphs to characterize connected claw-free graphs with minimum degree at least three that have equal total domination and matching numbers.
\end{abstract}

Keywords: claw-free, matching number, total domination number

\section{Introduction}

Total domination in graphs was introduced by Cockayne, Dawes, and Hedetniemi [3] and is now well studied in graph theory. The literature on this subject has been surveyed and detailed in the two books by Haynes, Hedetniemi, and Slater [5, 6].

\footnotetext{
${ }^{*}$ Research supported in part by the South African National Research Foundation and the University of KwaZulu-Natal.
} 
Let $G=(V, E)$ be a graph with vertex set $V$ and edge set $E$. A set $S \subseteq V$ is a total dominating set, abbreviated TDS, of $G$ if every vertex in $V$ is adjacent to a vertex in $S$. Every graph without isolated vertices has a TDS, since $S=V$ is such a set. The total domination number of $G$, denoted by $\gamma_{t}(G)$, is the minimum cardinality of a TDS of $G$. A TDS of $G$ of cardinality $\gamma_{t}(G)$ is called a $\gamma_{t}(G)$-set.

Two edges in a graph $G$ are independent if they are not adjacent in $G$. A set of pairwise independent edges of $G$ is called a matching in $G$, while a matching of maximum cardinality is a maximum matching. The number of edges in a maximum matching of $G$ is called the matching number of $G$ which we denote by $\alpha^{\prime}(G)$. A perfect matching in $G$ is a matching with the property that every vertex is incident with an edge of the matching. Matchings in graphs are extensively studied in the literature (see, for example, the survey articles by Plummer [10] and Pulleyblank [11]).

For notation and graph theory terminology we in general follow [5]. Specifically, let $G=(V, E)$ be a graph with vertex set $V$ of order $n(G)=|V|$ and edge set $E$ of size $m(G)=$ $|E|$, and let $v$ be a vertex in $V$. The open neighborhood of $v$ in $G$ is $N(v)=\{u \in V \mid u v \in$ $E\}$, and its closed neighborhood is the set $N[v]=N(v) \cup\{v\}$. For a set $S \subseteq V$, its open neighborhood is the set $N(S)=\cup_{v \in S} N(v)$ and its closed neighborhood is the set $N[S]=N(S) \cup S$. If $Y \subseteq V$, then the set $S$ is said to dominate the set $Y$ if $Y \subseteq N[S]$, while $S$ totally dominates $Y$ if $Y \subseteq N(S)$.

Throughout this paper, we only consider finite, simple undirected graphs without isolated vertices. For a subset $S \subseteq V$, the subgraph induced by $S$ is denoted by $G[S]$. A vertex of degree $k$ we call a degree- $k$ vertex. We denote the minimum degree of the graph $G$ by $\delta(G)$ and its maximum degree by $\Delta(G)$. A graph $G$ is claw-free if it has no induced subgraph isomorphic to $K_{1,3}$. A graph is cubic if every vertex has degree 3 , while we say that a graph is almost cubic if it has one vertex of degree 4 and all other vertices of degree 3 .

The transversal number $\tau(H)$ of a hypergraph $H$ is the minimum number of vertices meeting every edge. For a graph $G=(V, E)$, we denote by $H_{G}$ the open neighborhood hypergraph, abbreviated $\mathrm{ONH}$, of $G$; that is, $H_{G}$ is the hypergraph with vertex set $V\left(H_{G}\right)=V$ and with edge set $E\left(H_{G}\right)=\left\{N_{G}(x) \mid x \in V(G)\right\}$ consisting of the open neighborhoods of vertices of $V$ in $G$. We observe that $\gamma_{t}(G)=\tau\left(H_{G}\right)$.

A hypergraph $H$ is said to be $k$-uniform if every edge of $H$ has size $k$. We call an edge of $H$ that contains $\ell$ vertices an $\ell$-edge. If $H$ has vertex set $V$ and $X \subseteq V$, we denote by $H \backslash X$ the induced subhypergraph on $V \backslash X$; that is, we delete all the vertices of $X$, and all the edges having a vertex in $X$. We denote the degree of $v$ in a hypergraph $H$ by $d_{H}(v)$, or simply by $d(v)$ if $H$ is clear from context. The hypergraph $H$ is said to be regular if every vertex of $H$ has the same degree. 


\section{Known Hypergraph Results}

\subsection{Hypergraph Results}

Chvátal and McDiarmid [2] and Tuza [15] independently established the following result about transversals in hypergraphs (see also [14] for a short proof of this result).

Theorem 1 ([2, 15]) If $H$ is a hypergraph on $n$ vertices and $m$ edges with all edges of size at least three, then $4 \tau(H) \leq n+m$.

We shall need the following definition.

Definition 1 Let $i, j \geq 0$ be arbitrary integers. Let $H_{i, j}^{4 e d g e}$ be the hypergraph defined as

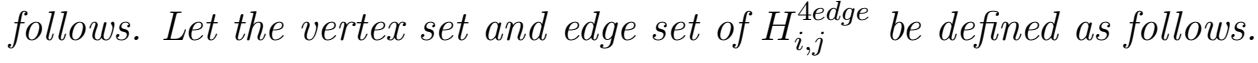

$$
\begin{aligned}
V\left(H_{i, j}^{\text {4edge }}\right) & =\left\{u, x_{0}, x_{1}, \ldots, x_{i}, y_{0}, y_{1}, \ldots, y_{i}, w_{0}, w_{1}, \ldots, w_{j}, z_{0}, z_{1}, \ldots, z_{j}\right\}, \\
E_{1} & =\bigcup_{a=1}^{i}\left\{\left\{x_{a-1}, x_{a}, y_{a}\right\},\left\{y_{a-1}, x_{a}, y_{a}\right\}\right\}, \\
E_{2} & \left.=\bigcup_{b=1}^{j}\left\{w_{b-1}, w_{b}, z_{b}\right\},\left\{z_{b-1}, w_{b}, z_{b}\right\}\right\}, \\
E\left(H_{i, j}^{\text {4edge }}\right) & =\left\{\left\{u, x_{0}, y_{0}\right\},\left\{u, w_{0}, z_{0}\right\},\left\{x_{0}, y_{0}, z_{0}, w_{0}\right\}\right\} \cup E_{1} \cup E_{2} .
\end{aligned}
$$

Let

$$
H^{4 e d g e}=\bigcup_{i \geq 0} \bigcup_{j \geq 0}\left\{H_{i, j}^{4 e d g e}\right\}
$$

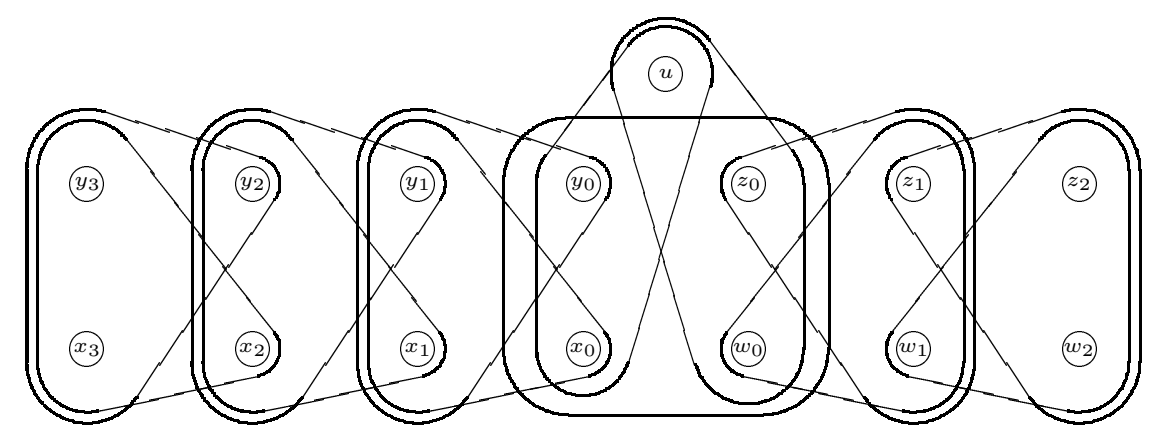

Figure 1: The hypergraph $H_{3,2}^{4 e d g e}$.

In Figure 1 we give an example of a hypergraph in the family $H^{4 e d g e}$.

We shall need the following result from [8].

Theorem 2 ([8]) Let $H$ be a connected hypergraph on $n$ vertices and $m$ edges where all edges contain at least three vertices. If $H$ is not 3-uniform and $4 \tau(H)=n+m$, then $H \in H^{4 e d g e}$. 


\subsection{Known Graph Results}

As an immediate consequence of Theorem 1, we have that the total domination number of a graph with minimum degree at least 3 is at most one-half its order.

Theorem 3 If $G$ is a graph of order $n$ with $\delta(G) \geq 3$, then $\gamma_{t}(G) \leq n / 2$.

Proof. The ONH hypergraph $H_{G}$ of $G$ has $n$ vertices and $n$ edges with all edges of size at least three. By Theorem 1, there exists a transversal in $H_{G}$ of size at most $(n+n) / 4=n / 2$. Hence, $\gamma_{t}(G)=\tau\left(H_{G}\right) \leq n / 2$.

We remark that Archdeacon et al. [1] recently found an elegant one page graph theoretic proof of Theorem 3 .

The connected claw-free cubic graphs achieving equality in Theorem 3 are characterized in [4] and contain at most eight vertices.

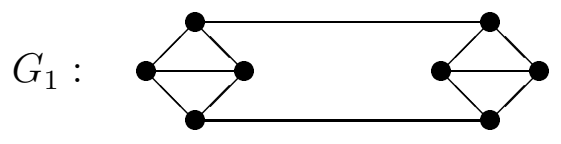

Figure 2: A claw-free cubic graph $G_{1}$ with $\gamma_{t}\left(G_{1}\right)=n / 2$.

Theorem 4 ([4]) If $G$ is a connected claw-free cubic graph of order $n$, then $\gamma_{t}(G) \leq\lfloor n / 2\rfloor$ with equality if and only if $G=K_{4}$ or $G=G_{1}$ where $G_{1}$ is the graph shown in Figure 2.

We now turn our attention to matchings in claw-free graphs. The following result was established independently by Las Vergnas [9] and Sumner [12, 13].

Theorem 5 ([9, 12, 13]) Every claw-free graph of even order has a perfect matching.

As a consequence of Theorem 5, we have the following result which was observed in [7].

Theorem 6 If $G$ is a claw-free graph of order $n$, then $\alpha^{\prime}(G)=\lfloor n / 2\rfloor$.

As a consequence of Theorems 3 and 6 , it follows that the total domination number of every claw-free graph with minimum degree at least three is bounded above by its matching number. This result was first observed in [7].

Theorem 7 ([7]) For every claw-free graph $G$ with $\delta(G) \geq 3, \gamma_{t}(G) \leq \alpha^{\prime}(G)$. 


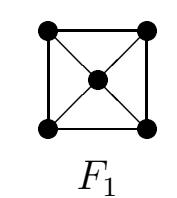

$F_{1}$
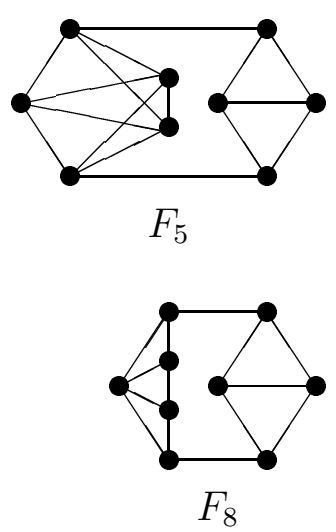
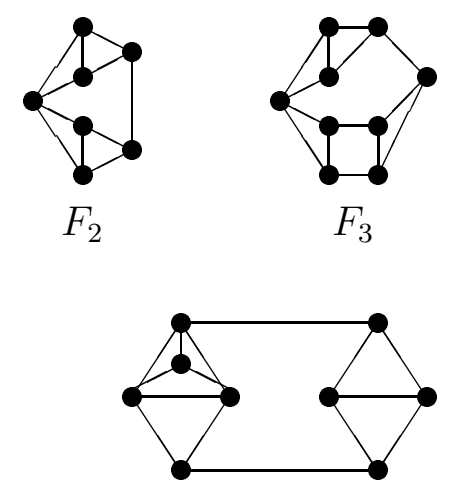

$F_{6}$

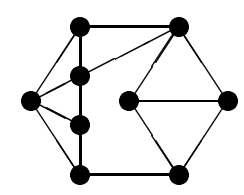

$F_{9}$
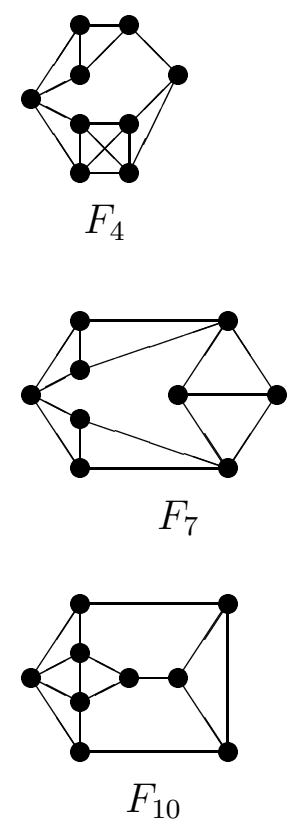

\section{Main Result}

Our aim in this paper is to characterize the connected claw-free graphs with minimum degree at least three that achieve equality in the bound of Theorem 7. For this purpose, we define a collection $\mathcal{F}$ of connected claw-free graphs with minimum degree three and maximum degree four that have equal total domination and matching numbers. Let $\mathcal{F}=\left\{F_{1}, F_{2}, \ldots, F_{12}\right\}$ be the collection of twelve graphs shown in Figure 3.

We shall prove:

Theorem 8 Let $G$ be a connected claw-free graph with $\delta(G) \geq 3$. Then, $\gamma_{t}(G)=\alpha^{\prime}(G)$ if and only if $G \in \mathcal{F} \cup\left\{K_{4}, K_{5}-e, K_{5}, G_{1}\right\}$.

\section{Proof of Theorem 8}

The sufficiency is straightforward to verify. As a consequence of Theorem 4, the graph $K_{4}$ and the graph $G_{1}$ of Figure 2 are the only connected claw-free cubic graphs that achieve equality in the bound of Theorem 7 . Hence it remains for us to characterize the
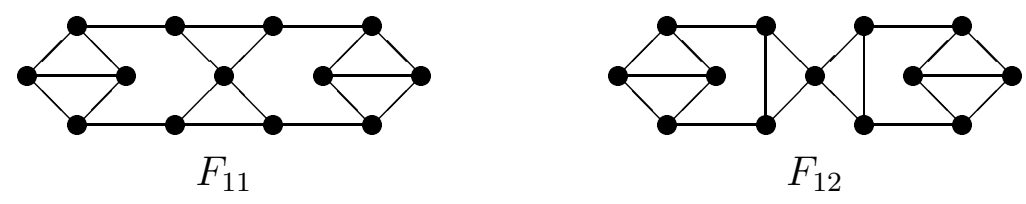

Figure 3: The collection $\mathcal{F}$ of twelve graphs. 
connected claw-free graphs with minimum degree at least three that are not cubic and achieve equality in the bound of Theorem 7 . We shall prove:

Theorem 9 If $G$ is a connected claw-free graph with minimum degree at least three and maximum degree at least four satisfying $\gamma_{t}(G)=\alpha^{\prime}(G)$, then $G \in \mathcal{F} \cup\left\{K_{5}-e, K_{5}\right\}$.

Proof. Let $G=(V, E)$ have order $n$. By Theorem $6, \alpha^{\prime}(G)=\lfloor n / 2\rfloor$. If $T$ is a transversal of $H_{G}$, then $|T| \geq \tau\left(H_{G}\right)=\gamma_{t}(G)=\alpha^{\prime}(G)$. Hence we have the following observation.

Observation 1 Every transversal in $H_{G}$ has size at least $\lfloor n / 2\rfloor$.

We shall frequently use the following observation, which is an application of Theorem 1.

Observation 2 If $V^{\prime} \subset V$ and $H^{\prime}=H_{G} \backslash V^{\prime}$ is a subhypergraph of $H_{G}$ of order $n^{\prime}$ and size $m^{\prime}$ in which every edge has size at least 3 , then there exists a transversal $T^{\prime}$ of $H^{\prime}$ such that $\left|T^{\prime}\right| \leq\left(m^{\prime}+n^{\prime}\right) / 4$.

Let $v$ be a vertex of maximum degree in $G$, and so $d(v)=\Delta(G) \geq 4$.

Observation $3 n$ is odd.

Proof. If $n$ is even, then in Observation 2, taking $V^{\prime}=\{v\}$, we have $n^{\prime}=n-1, m^{\prime} \leq n-4$ and $\left|T^{\prime}\right| \leq(2 n-5) / 4$. Thus, $T=T^{\prime} \cup\{v\}$ is a transversal of $H_{G}$ of size less than $\lfloor n / 2\rfloor$, contradicting Observation 1. Hence, $n$ is odd.

As a consequence of Theorem 2, we have the following observation.

Observation $4 \Delta(G)=4$.

Proof. Suppose that $\Delta(G) \geq 5$. Let $H^{\prime}=H_{G} \backslash\{v\}$. Then, $H^{\prime}$ has order $n^{\prime}=n-1$ and size $m^{\prime} \leq n-5$, and every edge of $H^{\prime}$ contains at least three vertices. Since $H^{\prime}$ contains the edge $N(v), H^{\prime}$ has at least one edge of size five or more. Hence, by Theorem 2, $\gamma_{t}(G) \leq \tau\left(H^{\prime}\right)+1 \leq\left(n^{\prime}+m^{\prime}-1\right) / 4+1 \leq(2 n-3) / 4$. The desired result now follows from the fact that $n$ is odd.

By Observations 3 and 4, $G$ contains an odd number of degree-4 vertices. Furthermore, by Theorem $6, \alpha^{\prime}(G)=(n-1) / 2$ and, by Observation 1 , every transversal in $H_{G}$ has size at least $(n-1) / 2$. As a consequence of Theorem 2 , we have the following result.

Observation 5 Every two degree-4 vertices in $G$ are at distance at most 2 apart.

Proof. Suppose that $G$ contains two degree- 4 vertices, say $u$ and $v$, at distance at least 3 apart. Let $H^{\prime}=H_{G} \backslash\{u, v\}$. Then, $H^{\prime}$ has order $n^{\prime}=n-2$ and size $m^{\prime}=n-8$, and every edge of $H^{\prime}$ is a 3-edge or a 4-edge. Further, $H^{\prime}$ has at least two 4-edges, namely $N(u)$ and $N(v)$. Let $H_{v}$ be the component of $H^{\prime}$ containing the 4-edge $N(v)$ (possibly, $\left.H_{v}=H^{\prime}\right)$. Let $N(v)=\left\{v_{1}, v_{2}, v_{3}, v_{4}\right\}$. 
Suppose $H_{v} \in H^{4 e d g e}$. Then, $H_{v}$ contains an edge $\left\{v_{1}, v_{2}, v_{5}\right\}$ containing $v_{1}$ and $v_{2}$, and an edge $\left\{v_{3}, v_{4}, v_{5}\right\}$ containing $v_{3}$ and $v_{4}$. Since the edges $N\left(v_{i}\right), 1 \leq i \leq 4$, are deleted from $H_{G}$ when constructing $H^{\prime}$, there must exist vertices $v_{6}$ and $v_{7}$ in $H_{v}$ such that in the graph $G, N\left(v_{6}\right)=\left\{v_{1}, v_{2}, v_{5}\right\}$ and $N\left(v_{7}\right)=\left\{v_{3}, v_{4}, v_{5}\right\}$. Thus in $G, v_{5} v_{6}$ and $v_{5} v_{7}$ are edges. Let $w \in N\left(v_{5}\right) \backslash\left\{v_{6}, v_{7}\right\}$. By the claw-freeness of $G$, we must have that $w v_{6}$ or $w v_{7}$ is an edge, implying that $w \in N(v)$. We may assume that $w=v_{1}$. Thus since $H_{v} \in H^{4 e d g e}$, $N\left(v_{5}\right)=\left\{v_{1}, v_{6}, v_{7}\right\}$ and there exists a vertex $v_{8}$ such that in $G, N\left(v_{8}\right)=\left\{v_{2}, v_{6}, v_{7}\right\}$. But then $d\left(v_{6}\right) \geq 4$, contradicting our earlier observation that $N\left(v_{6}\right)=\left\{v_{1}, v_{2}, v_{5}\right\}$. Hence, $H_{v} \notin H^{4 e d g e}$.

By Theorem 2, $4 \tau\left(H_{v}\right) \leq\left|V\left(H_{v}\right)\right|+\left|E\left(H_{v}\right)\right|-1$. Applying Theorems 1 and 2 to every other component of $H^{\prime}$, if any, it follows that $4 \tau\left(H^{\prime}\right) \leq n^{\prime}+m^{\prime}-1=2 n-11$. However if $T^{\prime}$ is a transversal of $H^{\prime}$, then $T^{\prime} \cup\{u, v\}$ is a TDS of $G$, and so $\gamma_{t}(G) \leq \tau\left(H^{\prime}\right)+2 \leq(2 n-3) / 4$, a contradiction.

Let $V=\left\{v, v_{1}, v_{2}, \ldots, v_{n-1}\right\}$. For $i=1,2, \ldots, n-1$, let $V_{i}=\left\{v_{1}, v_{2}, \ldots, v_{i}\right\}$. We may assume that $N(v)=V_{4}$. Let $G_{v}=G\left[V_{4}\right]$. If $n=5$, then $G_{v} \in\left\{C_{4}, K_{4}-e, K_{4}\right\}$ in which case $G \in\left\{F_{1}, K_{5}-e, K_{5}\right\}$ Hence we may assume that $n \geq 7$. Thus, $G_{v}$ contains at most five edges and, since $G$ is claw-free, $G_{v}$ contains at least two edges.

Observation 6 If $G_{v}=K_{4}-e$, then $G=F_{5}$.

Proof. We may assume that $v_{3} v_{4}$ is the edge missing in $G_{v}$ and that $d\left(v_{4}\right)=4$. If $d\left(v_{3}\right)=3$, then in Observation 2, taking $V^{\prime}=N[v]$, we have $n^{\prime}=n-5, m^{\prime}=n-6$ and $\left|T^{\prime}\right| \leq(2 n-11) / 4$. Thus, $T=T^{\prime} \cup\left\{v, v_{4}\right\}$ is a transversal of $H_{G}$ of size at most $(2 n-3) / 4$, contradicting Observation 1 . Hence, $d\left(v_{3}\right)=4$.

Let $G^{\prime}=G-v$. Then, $G^{\prime}$ is a claw-free graph with $\delta\left(G^{\prime}\right) \geq 3$ of even order $n^{\prime}=n-1$. If $\gamma_{t}\left(G^{\prime}\right)<n^{\prime} / 2$, then $\gamma_{t}\left(G^{\prime}\right) \leq\left(n^{\prime}-2\right) / 2$. However every TDS of $G^{\prime}$ contains a vertex from the set $\{2,3,4\}$ (in order to totally dominate $v_{1}$ ) and is therefore also a TDS of $G$, implying that $\gamma_{t}(G) \leq(n-3) / 2$, a contradiction. Hence, $\gamma_{t}\left(G^{\prime}\right) \geq n^{\prime} / 2$. Thus by Theorem $4, G^{\prime}=G_{1}$ and so $G=F_{5}$.

By Observation 6, we may assume that the subgraph induced by the neighborhood of every degree-4 vertex is not $K_{4}-e$.

Observation 7 The subgraph induced by the neighborhood of every degree-4 vertex is not a 4-cycle.

Proof. Suppose $G_{v}=C_{4}$. We may assume that $G_{v}$ is given by the cycle $v_{1}, v_{2}, v_{3}, v_{4}, v_{1}$. Since $n \geq 7$, we may assume that $d\left(v_{1}\right)=4$ and that $v_{1} v_{5} \in E(G)$. Since $G$ is claw-free, we may further assume that $v_{2} v_{5} \in E(G)$. If $v_{3} v_{5}$ or $v_{4} v_{5}$ is an edge, then $n=6$, a contradiction. Hence neither $v_{3} v_{5}$ nor $v_{4} v_{5}$ is an edge.

If $d\left(v_{3}\right)=3$, then in Observation 2, taking $V^{\prime}=N[v]$, we have $n^{\prime}=n-5, m^{\prime}=n-6$ and $\left|T^{\prime}\right| \leq(2 n-11) / 4$. Thus, $T=T^{\prime} \cup\left\{v_{1}, v_{4}\right\}$ is a transversal of $H_{G}$ of size at most $(2 n-3) / 4$, contradicting Observation 1. Hence, $d\left(v_{3}\right)=4$. In Observation 2, taking $V^{\prime}=V_{3} \cup\{v\}$, we have $n^{\prime}=n-4, m^{\prime}=n-7$ and $\left|T^{\prime}\right| \leq(2 n-11) / 4$. Thus, $T=T^{\prime} \cup\left\{v_{2}, v_{3}\right\}$ is a transversal of $H_{G}$ of size at most $(2 n-3) / 4$, contradicting Observation 1. 
Observation 8 If $G_{v}=K_{1} \cup C_{3}$, then $G=F_{6}$.

Proof. Suppose that $G_{v}=K_{1} \cup C_{3}$, where $v_{1}$ is the isolated vertex of $G_{v}$. If at least two vertices in $N(v) \backslash\left\{v_{1}\right\}$ have degree 3, say $v_{2}$ and $v_{3}$, then in Observation 2, taking $V^{\prime}=V_{3} \cup\{v\}$, we have $n^{\prime}=n-4, m^{\prime} \leq n-7$ and $\left|T^{\prime}\right| \leq(2 n-11) / 4$. Thus, $T=T^{\prime} \cup\left\{v, v_{1}\right\}$ is a transversal of $H_{G}$ of size at most $(2 n-3) / 4$, contradicting Observation 1 . Hence at most one vertex in $N(v) \backslash\left\{v_{1}\right\}$ has degree 3 . We proceed further with the following claim.

Claim 1 One vertex in $N(v) \backslash\left\{v_{1}\right\}$ has degree 3.

Proof. Suppose, to the contrary, that each vertex in $\left\{v_{2}, v_{3}, v_{4}\right\}$ has degree 4 . Let $\left\{v_{5}, v_{6}\right\} \subseteq N\left(v_{1}\right) \backslash\{v\}$. Then, $v_{5} v_{6}$ is an edge. Suppose there is an edge joining $\left\{v_{2}, v_{3}, v_{4}\right\}$ and $\left\{v_{5}, v_{6}\right\}$, say $v_{2} v_{5}$. Then in Observation 2, taking $V^{\prime}=V_{2} \cup\left\{v, v_{5}\right\}$, we have $n^{\prime}=n-4$, $m^{\prime} \leq n-7$ and $\left|T^{\prime}\right| \leq(2 n-11) / 4$. Thus, $T=T^{\prime} \cup\left\{v, v_{1}\right\}$ is a transversal of $H_{G}$ of size at most $(2 n-3) / 4$, contradicting Observation 1 . Hence, there is no edge joining $\left\{v_{2}, v_{3}, v_{4}\right\}$ and $\left\{v_{5}, v_{6}\right\}$. For $i=2,3,4$, let $N\left(v_{i}\right) \backslash N[v]=\left\{v_{i}^{\prime}\right\}$.

Case 1. $v_{i}^{\prime}=v_{j}^{\prime}$ for some $i$ and $j$, where $2 \leq i<j \leq 4$. We may assume that $i=2$ and $j=3$, and that $v_{7}=v_{2}^{\prime}$. If $v_{4}^{\prime}=v_{7}$, then we contradict our assumption that the subgraph induced by the neighborhood of every degree- 4 vertex is not $K_{4}-e$. Hence, $v_{4}^{\prime} \neq v_{7}$. We may assume that $v_{4}^{\prime}=v_{8}$, and so $N\left(v_{4}\right)=\left\{v, v_{2}, v_{3}, v_{8}\right\}$.

Suppose that $v_{7}$ is adjacent to $v_{5}$ or $v_{6}$, say $v_{5}$. If $d\left(v_{1}\right)=4$ or $d\left(v_{5}\right)=4$, then in Observation 2, taking $V^{\prime}=V_{3} \cup\left\{v, v_{5}, v_{7}\right\}$, we have $n^{\prime}=n-6, m^{\prime} \leq n-9$ and $\left|T^{\prime}\right| \leq$ $(2 n-15) / 4$. Thus, $T=T^{\prime} \cup\left\{v, v_{1}, v_{5}\right\}$ is a transversal of $H_{G}$ of size at most $(2 n-3) / 4$, contradicting Observation 1. Hence, $d\left(v_{1}\right)=d\left(v_{5}\right)=3$. If $v_{6} v_{7}$ is an edge, then taking $V^{\prime}=\left(V_{7} \backslash\left\{v_{4}\right\}\right) \cup\{v\}$, we have $n^{\prime}=n-7, m^{\prime}=n-8$ and $\left|T^{\prime}\right| \leq(2 n-15) / 4$. Thus, $T=T^{\prime} \cup\left\{v, v_{1}, v_{5}\right\}$ is a transversal of $H_{G}$ of size at most $(2 n-3) / 4$, contradicting Observation 1. Hence, $v_{6} v_{7}$ is not an edge, implying that $d\left(v_{7}\right)=3$. If $v_{6} v_{8}$ is not an edge, then in Observation 2, taking $V^{\prime}=V_{5} \cup\left\{v, v_{7}, v_{8}\right\}$, we have $n^{\prime}=n-8, m^{\prime} \leq n-11$ and $\left|T^{\prime}\right| \leq(2 n-19) / 4$. Thus, $T=T^{\prime} \cup\left\{v_{1}, v_{4}, v_{5}, v_{8}\right\}$ is a transversal of $H_{G}$ of size at most $(2 n-3) / 4$, contradicting Observation 1 . Hence, $v_{6} v_{8}$ is an edge. Therefore in Observation 2, taking $V^{\prime}=V_{8} \cup\{v\}$, we have $n^{\prime}=n-9, m^{\prime} \leq n-10$ and $\left|T^{\prime}\right| \leq(2 n-19) / 4$. Thus, $T=T^{\prime} \cup\left\{v_{1}, v_{4}, v_{5}, v_{8}\right\}$ is a transversal of $H_{G}$ of size at most $(2 n-3) / 4$, contradicting Observation 1. Hence, $v_{7}$ is adjacent to neither $v_{5}$ nor $v_{6}$.

Suppose that $v_{7} v_{8}$ is an edge. Let $v_{9}$ be the common neighbor of $v_{7}$ and $v_{8}$, which exists as $G$ is claw-free. In Observation 2, taking $V^{\prime}=V_{4} \cup\left\{v, v_{9}\right\}$, we have $n^{\prime}=n-6$, $m^{\prime} \leq n-9$ and $\left|T^{\prime}\right| \leq(2 n-15) / 4$. Thus, $T=T^{\prime} \cup\left\{v, v_{1}, v_{9}\right\}$ is a transversal of $H_{G}$ of size at most $(2 n-3) / 4$, contradicting Observation 1 . Hence, $v_{7} v_{8}$ is not an edge.

Suppose that $v_{8}$ is adjacent to $v_{5}$ or $v_{6}$, say $v_{5}$. In Observation 2, taking $V^{\prime}=V_{8} \cup\{v\}$, we have $n^{\prime}=n-9, m^{\prime} \leq n-10$ and $\left|T^{\prime}\right| \leq(2 n-19) / 4$. Thus, $T=T^{\prime} \cup\left\{v_{2}, v_{5}, v_{6}, v_{7}\right\}$ is a transversal of $H_{G}$ of size at most $(2 n-3) / 4$, contradicting Observation 1 . Hence, $v_{8}$ is adjacent to neither $v_{5}$ nor $v_{6}$.

Suppose that $v_{7}$ and $v_{8}$ have a common neighbor, say $v_{9}$. In Observation 2 , taking $V^{\prime}=V_{4} \cup\left\{v, v_{9}\right\}$, we have $n^{\prime}=n-6, m^{\prime} \leq n-9$ and $\left|T^{\prime}\right| \leq(2 n-15) / 4$. Thus, $T=T^{\prime} \cup\left\{v, v_{1}, v_{9}\right\}$ is a transversal of $H_{G}$ of size at most $(2 n-3) / 4$, contradicting 
Observation 1. Hence, $v_{7}$ and $v_{8}$ have no common neighbor. Let $v_{9} \in N\left(v_{7}\right) \backslash\left\{v_{2}, v_{3}\right\}$ and let $\left\{v_{10}, v_{11}\right\} \subseteq N\left(v_{8}\right)$.

Suppose that $v_{9}$ is adjacent to $v_{10}$ or $v_{11}$, say $v_{10}$. In Observation 2 , taking $V^{\prime}=$ $\left(V_{10} \backslash\left\{v_{5}, v_{6}\right\}\right) \cup\{v\}$, we have $n^{\prime}=n-9, m^{\prime} \leq n-12$ and $\left|T^{\prime}\right| \leq(2 n-21) / 4$. Thus, $T=T^{\prime} \cup\left\{v, v_{1}, v_{9}, v_{10}\right\}$ is a transversal of $H_{G}$ of size at most $(2 n-5) / 4$, contradicting Observation 1 . Hence, $v_{9}$ is adjacent to neither $v_{10}$ nor $v_{11}$. Suppose that $v_{9}$ is adjacent to $v_{5}$ or $v_{6}$, say $v_{5}$. In Observation 2 , taking $V^{\prime}=\left(V_{9} \backslash\left\{v_{6}\right\}\right) \cup\{v\}$, we have $n^{\prime}=n-9$, $m^{\prime} \leq n-12$ and $\left|T^{\prime}\right| \leq(2 n-21) / 4$. Thus, $T=T^{\prime} \cup\left\{v_{4}, v_{5}, v_{8}, v_{9}\right\}$ is a transversal of $H_{G}$ of size at most $(2 n-5) / 4$, contradicting Observation 1 . Hence, $v_{9}$ is adjacent to neither $v_{5}$ nor $v_{6}$. Thus in Observation 2, taking $V^{\prime}=\left(V_{9} \backslash\left\{v_{1}, v_{6}, v_{7}\right\}\right) \cup\{v\}$, we have $n^{\prime}=n-7$, $m^{\prime} \leq n-12$ and $\left|T^{\prime}\right| \leq(2 n-19) / 4$. Thus, $T=T^{\prime} \cup\left\{v_{4}, v_{5}, v_{8}, v_{9}\right\}$ is a transversal of $H_{G}$ of size at most $(2 n-3) / 4$, contradicting Observation 1 . We conclude that $v_{i}^{\prime} \neq v_{j}^{\prime}$ for $2 \leq i<j \leq 4$.

Case 2. $v_{i}^{\prime} \neq v_{j}^{\prime}$ for $2 \leq i<j \leq 4$. For $i \in\{2,3,4\}$, let $v_{i}^{\prime}=v_{i+5}$. Thus, $v_{2} v_{7}, v_{3} v_{8}$ and $v_{4} v_{9}$ are edges.

Suppose that there is an edge joining $\left\{v_{5}, v_{6}\right\}$ and $\left\{v_{7}, v_{8}, v_{9}\right\}$, say $v_{5} v_{7}$. If $v_{6} v_{7}$ is an edge, then in Observation 2, taking $V^{\prime}=\left(V_{8} \backslash\left\{v_{4}\right\}\right) \cup\{v\}$, we have $n^{\prime}=n-8, m^{\prime} \leq n-11$ and $\left|T^{\prime}\right| \leq(2 n-19) / 4$. Thus, $T=T^{\prime} \cup\left\{v_{3}, v_{5}, v_{7}, v_{8}\right\}$ is a transversal of $H_{G}$ of size at most $(2 n-3) / 4$, contradicting Observation 1 . Hence, $v_{6} v_{7}$ is not an edge. If $v_{8}$ or $v_{9}$, say $v_{8}$, is a common neighbor of $v_{5}$ and $v_{7}$, then in Observation 2, taking $V^{\prime}=\left(V_{9} \backslash\left\{v_{6}\right\}\right) \cup\{v\}$, we have $n^{\prime}=n-9, m^{\prime} \leq n-10$ and $\left|T^{\prime}\right| \leq(2 n-19) / 4$. Thus, $T=T^{\prime} \cup\left\{v_{1}, v_{4}, v_{5}, v_{9}\right\}$ is a transversal of $H_{G}$ of size at most $(2 n-3) / 4$, contradicting Observation 1 . Hence we may assume that $v_{10}$ is the common neighbor of $v_{5}$ and $v_{7}$. But then in Observation 2, taking $V^{\prime}=\left(V_{5} \backslash\left\{v_{1}\right\}\right) \cup\{v\}$, we have $n^{\prime}=n-5, m^{\prime} \leq n-10$ and $\left|T^{\prime}\right| \leq(2 n-15) / 4$. Thus, $T=T^{\prime} \cup\left\{v_{3}, v_{4}, v_{5}\right\}$ is a transversal of $H_{G}$ of size at most $(2 n-3) / 4$, contradicting Observation 1. Hence there is no edge joining $\left\{v_{5}, v_{6}\right\}$ and $\left\{v_{7}, v_{8}, v_{9}\right\}$.

Suppose that $\left\{v_{7}, v_{8}, v_{9}\right\}$ is not an independent set. We may assume that $v_{7} v_{8}$ is an edge. Then in Observation 2, taking $V^{\prime}=\left(V_{8} \backslash\left\{v_{1}, v_{4}, v_{6}\right\}\right) \cup\{v\}$, we have $n^{\prime}=n-6$, $m^{\prime} \leq n-10$ and $\left|T^{\prime}\right| \leq(2 n-16) / 4$. Thus, $T=T^{\prime} \cup\left\{v_{2}, v_{5}, v_{7}\right\}$ is a transversal of $H_{G}$ of size at most $(2 n-4) / 4$, contradicting Observation 1. Hence, $\left\{v_{7}, v_{8}, v_{9}\right\}$ is an independent set.

Suppose that two vertices in $\left\{v_{7}, v_{8}, v_{9}\right\}$ have a common neighbor. We may assume that $v_{7}$ and $v_{8}$ have a common neighbor, say $v_{10}$. Then in Observation 2, taking $V^{\prime}=V_{3} \cup$ $\left\{v, v_{10}\right\}$, we have $n^{\prime}=n-5, m^{\prime} \leq n-10$ and $\left|T^{\prime}\right| \leq(2 n-15) / 4$. Thus, $T=T^{\prime} \cup\left\{v, v_{1}, v_{10}\right\}$ is a transversal of $H_{G}$ of size at most $(2 n-3) / 4$, contradicting Observation 1 . Hence no two vertices in $\left\{v_{7}, v_{8}, v_{9}\right\}$ have a common neighbor. Let $\left\{v_{10}, v_{11}\right\} \subseteq N\left(v_{7}\right),\left\{v_{12}, v_{13}\right\} \subseteq N\left(v_{8}\right)$ and $\left\{v_{14}, v_{15}\right\} \subseteq N\left(v_{9}\right)$. Then, $v_{10} v_{11}, v_{12} v_{13}$ and $v_{14} v_{15}$ are all edges.

Suppose there is an edge joining two triangles each of which contain a vertex from $\left\{v_{10}, v_{11}, v_{12}, v_{13}, v_{14}, v_{15}\right\}$. We may assume that $v_{10} v_{12}$ is an edge. Then in Observation 2, taking $V^{\prime}=V_{3} \cup\left\{v, v_{7}, v_{8}, v_{10}, v_{12}\right\}$, we have $n^{\prime}=n-8, m^{\prime} \leq n-13$ and $\left|T^{\prime}\right| \leq$ $(2 n-21) / 4$. Thus, $T=T^{\prime} \cup\left\{v, v_{1}, v_{10}, v_{12}\right\}$ is a transversal of $H_{G}$ of size at most $(2 n-5) / 4$, contradicting Observation 1. Hence there is no edge joining two triangles each of which contain a vertex from $\left\{v_{10}, v_{11}, v_{12}, v_{13}, v_{14}, v_{15}\right\}$. 
Suppose there is an edge joining $\left\{v_{5}, v_{6}\right\}$ and $\left\{v_{10}, v_{11}, v_{12}, v_{13}, v_{14}, v_{15}\right\}$, say $v_{5} v_{10}$. Then in Observation 2, taking $V^{\prime}=\left(V_{8} \backslash\left\{v_{4}\right\}\right) \cup\left\{v, v_{10}, v_{14}\right\}$, we have $n^{\prime}=n-10, m^{\prime} \leq n-15$ and $\left|T^{\prime}\right| \leq(2 n-25) / 4$. Thus, $T=T^{\prime} \cup\left\{v_{3}, v_{5}, v_{8}, v_{10}, v_{14}\right\}$ is a transversal of $H_{G}$ of size at most $(2 n-5) / 4$, contradicting Observation 1 . Hence there is no edge joining $\left\{v_{5}, v_{6}\right\}$ and $\left\{v_{10}, v_{11}, v_{12}, v_{13}, v_{14}, v_{15}\right\}$. Then in Observation 2, taking $V^{\prime}=V_{4} \cup\left\{v, v_{10}, v_{12}, v_{14}\right\}$, we have $n^{\prime}=n-8, m^{\prime} \leq n-15$ and $\left|T^{\prime}\right| \leq(2 n-23) / 4$. Thus, $T=T^{\prime} \cup\left\{v, v_{1}, v_{10}, v_{12}, v_{14}\right\}$ is a transversal of $H_{G}$ of size at most $(2 n-3) / 4$, contradicting Observation 1. This completes the proof of Claim 1.

By Claim 1, one vertex in $N(v) \backslash\left\{v_{1}\right\}$ has degree 3. We may assume that $d\left(v_{2}\right)=3$. Then, $d\left(v_{3}\right)=d\left(v_{4}\right)=4$. If $d\left(v_{1}\right)=4$, then in Observation 2, taking $V^{\prime}=V_{2} \cup\{v\}$, we have $n^{\prime}=n-3, m^{\prime}=n-8$ and $\left|T^{\prime}\right| \leq(2 n-11) / 4$. Thus, $T=T^{\prime} \cup\left\{v, v_{1}\right\}$ is a transversal of $H_{G}$ of size at most $(2 n-3) / 4$, contradicting Observation 1 . Hence, $d\left(v_{1}\right)=3$. We may assume that $N\left(v_{1}\right)=\left\{v, v_{5}, v_{6}\right\}$. Thus, $v_{5} v_{6}$ is an edge.

Suppose there is an edge joining $\left\{v_{3}, v_{4}\right\}$ and $\left\{v_{5}, v_{6}\right\}$, say $v_{3} v_{5}$. Then in Observation 2, taking $V^{\prime}=V_{3} \cup\{v\}$, we have $n^{\prime}=n-4, m^{\prime}=n-7$ and $\left|T^{\prime}\right| \leq(2 n-11) / 4$. Thus, $T=$ $T^{\prime} \cup\left\{v, v_{1}\right\}$ is a transversal of $H_{G}$ of size at most $(2 n-3) / 4$, contradicting Observation 1 . Hence, there is no edge joining $\left\{v_{3}, v_{4}\right\}$ and $\left\{v_{5}, v_{6}\right\}$. Let $N\left(v_{3}\right)=\left\{v, v_{2}, v_{4}, v_{7}\right\}$.

Claim $2 \quad v_{4} v_{7}$ is an edge.

Proof. Suppose, to the contrary, that $v_{4} v_{7}$ is not an edge. Let $N\left(v_{4}\right)=\left\{v, v_{2}, v_{3}, v_{8}\right\}$. Suppose there is an edge joining $\left\{v_{5}, v_{6}\right\}$ and $\left\{v_{7}, v_{8}\right\}$, say $v_{5} v_{7}$. If $v_{6} v_{7}$ is an edge, then in Observation 2, taking $V^{\prime}=\left(V_{7} \backslash\left\{v_{4}\right\}\right) \cup\{v\}$, we have $n^{\prime}=n-7, m^{\prime} \leq n-8$ and $\left|T^{\prime}\right| \leq(2 n-15) / 4$. If $v_{6} v_{7}$ is not an edge, then there is a common neighbor of $v_{5}$ and $v_{7}$ (which may possibly be $v_{8}$ ), and in Observation 2, taking $V^{\prime}=V_{3} \cup\left\{v, v_{5}, v_{7}\right\}$, we have $n^{\prime}=n-6, m^{\prime}=n-9$ and $\left|T^{\prime}\right| \leq(2 n-15) / 4$. In both cases, $T=T^{\prime} \cup\left\{v, v_{1}, v_{5}\right\}$ is a transversal of $H_{G}$ of size at most $(2 n-3) / 4$, contradicting Observation 1. Hence, there is no edge joining $\left\{v_{5}, v_{6}\right\}$ and $\left\{v_{7}, v_{8}\right\}$.

Since each of $v_{5}$ and $v_{6}$ is at distance 3 from a degree- 4 vertex (namely, $v_{3}$ and $v_{4}$ ), $d\left(v_{5}\right)=d\left(v_{6}\right)=3$ by Observation 5. Further for $i \geq 9, d\left(v, v_{i}\right) \geq 3$, and so, by Observation $5, d\left(v_{i}\right)=3$.

Suppose that $v_{7} v_{8}$ is an edge. Let $v_{9}$ be a common neighbor of $v_{7}$ and $v_{8}$. In Observation 2, taking $V^{\prime}=V_{4} \cup\left\{v, v_{9}\right\}$, we have $n^{\prime}=n-6, m^{\prime} \leq n-9$ and $\left|T^{\prime}\right| \leq(2 n-15) / 4$. Thus, $T=T^{\prime} \cup\left\{v, v_{1}, v_{9}\right\}$ is a transversal of $H_{G}$ of size at most $(2 n-3) / 4$, contradicting Observation 1 . Hence, $v_{7} v_{8}$ is not an edge.

Suppose $d\left(v_{7}\right)=4$. Then $N\left[v_{7}\right] \backslash\left\{v_{3}\right\}$ induces a clique $K_{4}$. Let $v_{7}^{\prime} \in N\left[v_{7}\right] \backslash\left\{v_{3}\right\}$. Then, $N\left[v_{7}^{\prime}\right]=N\left[v_{7}\right] \backslash\left\{v_{3}\right\}$. In Observation 2, taking $V^{\prime}=N[v] \cup N\left[v_{7}\right]$, we have $n^{\prime}=n-9$, $m^{\prime}=n-11$ and $\left|T^{\prime}\right| \leq(2 n-20) / 4$. Thus, $T=T^{\prime} \cup\left\{v, v_{1}, v_{7}, v_{7}^{\prime}\right\}$ is a transversal of $H_{G}$ of size at most $(2 n-4) / 4$, contradicting Observation 1 . Hence, $d\left(v_{7}\right)=3$. Similarly, $d\left(v_{8}\right)=3$.

Let $N\left(v_{7}\right)=\left\{v_{3}, v_{9}, v_{10}\right\}$. Then, $v_{9} v_{10}$ is an edge. Suppose $v_{8}$ is adjacent to $v_{9}$ or $v_{10}$, say $v_{9}$. Then, $N\left(v_{9}\right)=\left\{v_{7}, v_{8}, v_{10}\right\}$. By the claw-freeness of $G, v_{8} v_{10}$ is an edge and $N\left(v_{8}\right)=\left\{v_{4}, v_{9}, v_{10}\right\}$. In Observation 2, taking $V^{\prime}=V_{4} \cup\left\{v, v_{7}, v_{8}, v_{9}, v_{10}\right\}$, we have 
$n^{\prime}=n-9, m^{\prime}=n-11$ and $\left|T^{\prime}\right| \leq(2 n-20) / 4$. Thus, $T=T^{\prime} \cup\left\{v, v_{1}, v_{7}, v_{9}\right\}$ is a transversal of $H_{G}$ of size at most $(2 n-4) / 4$, contradicting Observation 1. Hence, $v_{8}$ is adjacent to neither $v_{9}$ nor $v_{10}$. Let $N\left(v_{8}\right)=\left\{v_{3}, v_{11}, v_{12}\right\}$. Then, $v_{11} v_{12}$ is an edge.

Suppose that there is an edge joining $\left\{v_{5}, v_{6}\right\}$ and $\left\{v_{9}, v_{10}, v_{11}, v_{12}\right\}$, say $v_{5} v_{9}$. In Observation 2, taking $V^{\prime}=V_{5} \cup\left\{v, v_{7}, v_{9}\right\}$, we have $n^{\prime}=n-8, m^{\prime}=n-11$ and $\left|T^{\prime}\right| \leq(2 n-19) / 4$. Thus, $T=T^{\prime} \cup\left\{v, v_{4}, v_{5}, v_{9}\right\}$ is a transversal of $H_{G}$ of size at most $(2 n-3) / 4$, contradicting Observation 1 . Hence, there is no edge joining $\left\{v_{5}, v_{6}\right\}$ and $\left\{v_{9}, v_{10}, v_{11}, v_{12}\right\}$.

Suppose that there is an edge joining $\left\{v_{9}, v_{10}\right\}$ and $\left\{v_{11}, v_{12}\right\}$, say $v_{9} v_{11}$. In Observation 2, taking $V^{\prime}=V_{4} \cup\left\{v, v_{7}, v_{8}, v_{9}, v_{11}\right\}$, we have $n^{\prime}=n-9, m^{\prime}=n-13$ and $\left|T^{\prime}\right| \leq(2 n-22) / 4$. Thus, $T=T^{\prime} \cup\left\{v, v_{1}, v_{9}, v_{11}\right\}$ is a transversal of $H_{G}$ of size at most $(2 n-6) / 4$, contradicting Observation 1 . Hence, there is no edge joining $\left\{v_{9}, v_{10}\right\}$ and $\left\{v_{11}, v_{12}\right\}$. Thus in Observation 2, taking $V^{\prime}=V_{4} \cup\left\{v, v_{9}, v_{11}\right\}$, we have $n^{\prime}=n-7$, $m^{\prime} \leq n-12$ and $\left|T^{\prime}\right| \leq(2 n-19) / 4$. Thus, $T=T^{\prime} \cup\left\{v, v_{1}, v_{9}, v_{11}\right\}$ is a transversal of $H_{G}$ of size at most $(2 n-3) / 4$, contradicting Observation 1 . This completes the proof of Claim 2.

By Claim 2, $v_{4} v_{7}$ is an edge. If $v_{7}$ is adjacent to $v_{5}$ or $v_{6}$, say $v_{5} v_{7}$, then in Observation 2, taking $V^{\prime}=V_{5} \cup\left\{v, v_{7}\right\}$, we have $n^{\prime}=n-7, m^{\prime} \leq n-8$ and $\left|T^{\prime}\right| \leq(2 n-15) / 4$. Thus, $T=T^{\prime} \cup\left\{v, v_{1}, v_{5}\right\}$ is a transversal of $H_{G}$ of size at most $(2 n-3) / 4$, contradicting Observation 1. Hence, $v_{7}$ is adjacent to neither $v_{5}$ nor $v_{6}$. Thus each of $v_{5}$ and $v_{6}$ is at distance 3 from a degree- 4 vertex (namely, $v_{3}$ and $v_{4}$ ), and so $d\left(v_{5}\right)=d\left(v_{6}\right)=3$ by Observation 5 .

Let $v_{8} \in N\left(v_{7}\right) \backslash\left\{v_{3}, v_{4}\right\}$. If $N\left(v_{8}\right) \neq\left\{v_{5}, v_{6}, v_{7}\right\}$, then in Observation 2, taking $V^{\prime}=V_{4} \cup\left\{v, v_{8}\right\}$, we have $n^{\prime}=n-6, m^{\prime} \leq n-9$ and $\left|T^{\prime}\right| \leq(2 n-15) / 4$. Thus, $T=T^{\prime} \cup\left\{v, v_{1}, v_{8}\right\}$ is a transversal of $H_{G}$ of size at most $(2 n-3) / 4$, contradicting Observation 1 . Hence, $N\left(v_{8}\right)=\left\{v_{5}, v_{6}, v_{7}\right\}$, implying that $G=F_{6}$. This completes the proof of Observation 8.

By Observation 8, we may assume that the subgraph induced by the neighborhood of every degree- 4 vertex is not $K_{1} \cup C_{3}$.

Observation 9 If $G_{v}=K_{1,3}+e$, then $G=F_{4}$.

Proof. We may assume that $v_{1}$ is the degree- 1 vertex in $G_{v}$ and that $v_{1} v_{2}$ is an edge. Thus, $v_{2}, v_{3}, v_{4}, v_{2}$ is a cycle. If $d\left(v_{3}\right)=d\left(v_{4}\right)=3$, then in Observation 2, taking $V^{\prime}=V_{4} \cup\{v\}$, we have $n^{\prime}=n-5, m^{\prime} \leq n-6$ and $\left|T^{\prime}\right| \leq(2 n-11) / 4$. Thus, $T=T^{\prime} \cup\left\{v, v_{1}\right\}$ is a transversal of $H_{G}$ of size at most $(2 n-3) / 4$, contradicting Observation 1 . Hence, we may assume that $d\left(v_{3}\right)=4$. Let $N\left(v_{3}\right)=\left\{v, v_{2}, v_{4}, v_{5}\right\}$.

If $v_{1} v_{5}$ is an edge, then in Observation 2, taking $V^{\prime}=V_{3} \cup\left\{v, v_{5}\right\}$, we have $n^{\prime}=n-5$, $m^{\prime} \leq n-6$ and $\left|T^{\prime}\right| \leq(2 n-11) / 4$. Thus, $T=T^{\prime} \cup\left\{v, v_{1}\right\}$ is a transversal of $H_{G}$ of size at most $(2 n-3) / 4$, contradicting Observation 1 . Hence, $v_{1} v_{5}$ is not an edge. But then $v_{4} v_{5}$ must be an edge, for otherwise $G\left[N\left(v_{3}\right)\right]=K_{1} \cup C_{3}$, contrary to assumption. Let $v_{6} \in N\left(v_{5}\right) \backslash\left\{v_{3}, v_{4}\right\}$. 
Suppose that $v_{1}$ and $v_{5}$ have a common neighbor. We may assume that $v_{1} v_{6}$ is an edge. Then in Observation 2, taking $V^{\prime}=V_{6} \cup\{v\}$, we have $n^{\prime}=n-7, m^{\prime} \leq n-8$ and $\left|T^{\prime}\right| \leq(2 n-15) / 4$. Thus, $T=T^{\prime} \cup\left\{v, v_{1}, v_{6}\right\}$ is a transversal of $H_{G}$ of size at most $(2 n-3) / 4$, contradicting Observation 1 . Hence, $v_{1}$ and $v_{5}$ have no common neighbor. In particular, $v_{1} v_{6}$ is not an edge. Thus, $d\left(v, v_{6}\right)=3$, and so, by Observation $5, d\left(v_{6}\right)=3$. Let $v_{7} \in N\left(v_{1}\right) \backslash\left\{v, v_{2}\right\}$. Then, $v_{5} v_{7}$ is not an edge. Thus, $d\left(v_{3}, v_{7}\right)=3$, and so, by Observation $5, d\left(v_{7}\right)=3$.

If $v_{6} v_{7}$ is not an edge, then in Observation 2, taking $V^{\prime}=V_{4} \cup\left\{v, v_{6}\right\}$, we have $n^{\prime}=n-6, m^{\prime} \leq n-9$ and $\left|T^{\prime}\right| \leq(2 n-15) / 4$. Thus, $T=T^{\prime} \cup\left\{v, v_{1}, v_{6}\right\}$ is a transversal of $H_{G}$ of size at most $(2 n-3) / 4$, contradicting Observation 1. Hence, $v_{6} v_{7}$ is an edge.

Suppose that $d\left(v_{1}\right)=d\left(v_{5}\right)=3$. Then, $v_{6}$ and $v_{7}$ have a common neighbor, $v_{8}$ say. In Observation 2, taking $V^{\prime}=V_{5} \cup\left\{v, v_{8}\right\}$, we have $n^{\prime}=n-7, m^{\prime} \leq n-9$ and $\left|T^{\prime}\right| \leq(2 n-16) / 4$. Thus, $T=T^{\prime} \cup\left\{v, v_{4}, v_{8}\right\}$ is a transversal of $H_{G}$ of size at most $(2 n-4) / 4$, contradicting Observation 1 . Hence, by symmetry, we may assume that $d\left(v_{1}\right)=4$. Let $N\left(v_{1}\right)=\left\{v, v_{2}, v_{7}, v_{8}\right\}$. Then, $v_{7} v_{8}$ is an edge. As shown with the vertex $v_{7}$, we must have that $v_{6} v_{8}$ is an edges and $d\left(v_{8}\right)=3$. But then, $G=F_{4}$.

By Observation 9, we may assume that the subgraph induced by the neighborhood of every degree- 4 vertex is not $K_{1,3}+e$.

Observation 10 If $G_{v}=P_{4}$, then $G \in\left\{F_{8}, F_{9}, F_{10}\right\}$.

Proof. We may assume that $G_{v}$ is given by the path $v_{1}, v_{2}, v_{3}, v_{4}$. We desired result now follows from Claim 3 and Claim 4.

Claim 3 If $d\left(v_{2}\right)=d\left(v_{3}\right)=3$, then $G=F_{8}$.

Proof. Suppose that $v_{1}$ or $v_{4}$ has degree 4 . We may assume that $d\left(v_{1}\right)=4$. In Observation 2, taking $V^{\prime}=V_{3} \cup\{v\}$, we have $n^{\prime}=n-4, m^{\prime} \leq n-7$ and $\left|T^{\prime}\right| \leq(2 n-11) / 4$. Thus, $T=T^{\prime} \cup\left\{v, v_{1}\right\}$ is a transversal of $H_{G}$ of size at most $(2 n-3) / 4$, contradicting Observation 1. Hence, $d\left(v_{1}\right)=d\left(v_{4}\right)=3$. Thus, since $G$ is claw-free, $v_{1}$ and $v_{4}$ have no common neighbor other than $v$. Let $N\left(v_{1}\right)=\left\{v, v_{2}, v_{5}\right\}$ and $N\left(v_{4}\right)=\left\{v, v_{3}, v_{6}\right\}$. For $i \geq 7$, the vertex $v_{i}$ is at distance at least 3 from the degree- 4 vertex $v$, and so, by Observation 5 , $d\left(v_{i}\right)=3$.

If $v_{5} v_{6}$ is an edge, then in Observation 2, taking $V^{\prime}=V_{6} \cup\{v\}$, we have $n^{\prime}=n-7$, $m^{\prime} \leq n-8$ and $\left|T^{\prime}\right| \leq(2 n-15) / 4$. Thus, $T=T^{\prime} \cup\left\{v, v_{1}, v_{5}\right\}$ is a transversal of $H_{G}$ of size at most $(2 n-3) / 4$, contradicting Observation 1 . Hence, $v_{5} v_{6}$ is not an edge.

By our assumption that the subgraph induced by the neighborhood of every degree- 4 vertex is not $K_{1} \cup C_{3}$, we have that $d\left(v_{5}\right)=d\left(v_{6}\right)=3$. Let $N\left(v_{5}\right)=\left\{v_{1}, v_{7}, v_{8}\right\}$. Then, $v_{7} v_{8} \in E$. If $v_{6} v_{7}$ is not an edge, then in Observation 2, taking $V^{\prime}=N[v] \cup\left\{v_{7}\right\}$, we have $n^{\prime}=n-6, m^{\prime} \leq n-9$ and $\left|T^{\prime}\right| \leq(2 n-15) / 4$. Thus, $T=T^{\prime} \cup\left\{v, v_{4}, v_{7}\right\}$ is a transversal of $H_{G}$ of size at most $(2 n-3) / 4$, contradicting Observation 1 . Hence, $v_{6} v_{7}$ is an edge. Thus, by the claw-freeness of $G, v_{6} v_{8}$ is an edge. Thus, $G=F_{8}$.

Claim 4 If $v_{2}$ or $v_{3}$ has degree 4 , then $G \in\left\{F_{9}, F_{10}\right\}$. 
Proof. We may assume that $d\left(v_{2}\right)=4$. Let $N\left(v_{2}\right)=\left\{v, v_{1}, v_{3}, v_{5}\right\}$. Since $G$ is claw-free, $v_{1} v_{5}$ or $v_{3} v_{5}$ is an edge. We consider two cases.

Case 1. $v_{3} v_{5}$ is an edge. Then, $v_{1} v_{5}$ is not an edge, for otherwise, $N\left(v_{2}\right)$ induces a 4cycle, contradicting Observation 7 . Similarly, $v_{4} v_{5}$ is not an edge. Let $v_{6} \in N\left(v_{5}\right) \backslash\left\{v_{2}, v_{3}\right\}$.

Case 1.1. $v_{5}$ has a common neighbor with $v_{1}$ or with $v_{4}$ that does not belong to $N(v)$. We may assume that $v_{1} v_{6}$ is an edge. Suppose that $d\left(v_{6}\right)=4$. Let $v_{7} \in N\left(v_{6}\right) \backslash\left\{v_{1}, v_{5}\right\}$. On the one hand, if $v_{4} v_{7}$ is not an edge, then in Observation 2, taking $V^{\prime}=V_{3} \cup\left\{v, v_{5}, v_{6}\right\}$, we have $n^{\prime}=n-6, m^{\prime} \leq n-9$ and $\left|T^{\prime}\right| \leq(2 n-15) / 4$. On the other hand, if $v_{4} v_{7}$ is an edge, then in Observation 2, taking $V^{\prime}=V_{6} \cup\{v\}$, we have $n^{\prime}=n-7, m^{\prime} \leq n-8$ and $\left|T^{\prime}\right| \leq(2 n-15) / 4$. In both cases, $T=T^{\prime} \cup\left\{v, v_{1}, v_{6}\right\}$ is a transversal of $H_{G}$ of size at most $(2 n-3) / 4$, contradicting Observation 1. Hence, $d\left(v_{6}\right)=3$ and $N\left(v_{6}\right)=\left\{v_{1}, v_{5}, v_{7}\right\}$. Then, $v_{1} v_{7}$ or $v_{5} v_{7}$ is an edge.

If $v_{1} v_{7}$ and $v_{5} v_{7}$ are edges, then in Observation 2, taking $V^{\prime}=\left(V_{7} \backslash\left\{v_{4}\right\}\right) \cup\{v\}$, we have $n^{\prime}=n-7, m^{\prime} \leq n-8$ and $\left|T^{\prime}\right| \leq(2 n-15) / 4$. Thus, $T=T^{\prime} \cup\left\{v, v_{1}, v_{7}\right\}$ is a transversal of $H_{G}$ of size at most $(2 n-3) / 4$, contradicting Observation 1. Hence, either $v_{1} v_{7}$ or $v_{5} v_{7}$ is an edge (but not both).

Suppose $v_{5} v_{7}$ is an edge. Then, $d\left(v_{1}\right)=3$. If $v_{4} v_{7}$ is an edge, then in Observation 2, taking $V^{\prime}=V_{7} \cup\{v\}$, we have $n^{\prime}=n-8, m^{\prime} \leq n-8$ and $\left|T^{\prime}\right| \leq(2 n-16) / 4$. Thus, $T=T^{\prime} \cup\left\{v_{2}, v_{5}, v_{7}\right\}$ is a transversal of $H_{G}$ of size at most $(2 n-4) / 4$, contradicting Observation 1. Hence, $v_{4} v_{7}$ is not an edge. Thus, $d\left(v, v_{7}\right)=3$, and so by Observation 5 , $d\left(v_{7}\right)=3$. Let $N\left(v_{7}\right)=\left\{v_{5}, v_{6}, v_{8}\right\}$. In Observation 2, taking $V^{\prime}=V_{8} \cup\{v\}$, we have $n^{\prime}=n-9, m^{\prime} \leq n-10$ and $\left|T^{\prime}\right| \leq(2 n-19) / 4$. Thus, $T=T^{\prime} \cup\left\{v, v_{4}, v_{7}, v_{8}\right\}$ is a transversal of $H_{G}$ of size at most $(2 n-3) / 4$, contradicting Observation 1 . Hence, $v_{5} v_{7}$ is not an edge. Thus, $v_{1} v_{7}$ is an edge and $d\left(v_{5}\right)=3$.

If $v_{4} v_{7}$ is an edge, then in Observation 2, taking $V^{\prime}=V_{7} \cup\{v\}$, we have $n^{\prime}=n-8$, $m^{\prime} \leq n-8$ and $\left|T^{\prime}\right| \leq(2 n-16) / 4$. Thus, $T=T^{\prime} \cup\left\{v_{3}, v_{4}, v_{7}\right\}$ is a transversal of $H_{G}$ of size at most $(2 n-4) / 4$, contradicting Observation 1 . Hence, $v_{4} v_{7}$ is not an edge. If $d\left(v_{4}\right)=d\left(v_{7}\right)=3$, then since $G$ is claw-free, $v_{4}$ and $v_{7}$ have no common neighbor. Thus in Observation 2, taking $V^{\prime}=\left(V_{5} \backslash\left\{v_{1}\right\}\right) \cup\left\{v, v_{7}\right\}$, we have $n^{\prime}=n-6, m^{\prime} \leq n-9$ and $\left|T^{\prime}\right| \leq(2 n-13) / 4$. Thus, $T=T^{\prime} \cup\left\{v_{2}, v_{3}, v_{7}\right\}$ is a transversal of $H_{G}$ of size at most $(2 n-5) / 4$, contradicting Observation 1 .

Case 1.2. $v_{5}$ has no common neighbor with $v_{1}$ or with $v_{4}$ that does not belong to $N(v)$. In particular, neither $v_{1} v_{6}$ nor $v_{4} v_{6}$ is an edge. Thus, $d\left(v, v_{6}\right)=3$, and so, by Observation $5, d\left(v_{6}\right)=3$.

Case 1.2.1 $v_{6}$ has a common neighbor with $v_{1}$ or $v_{4}$. We may assume that $v_{1}$ and $v_{6}$ have a common neighbor, $v_{7}$ say. By Case 1.1, $v_{5} v_{7}$ is not an edge. By the claw-freeness of $G, v_{4} v_{7}$ is not an edge. Thus, $d\left(v_{3}, v_{7}\right)=3$, and so, by Observation $5, d\left(v_{7}\right)=3$. Let $N\left(v_{7}\right)=\left\{v_{1}, v_{6}, v_{8}\right\}$. Then, $v_{1} v_{8}$ or $v_{6} v_{8}$ is an edge. If both $v_{1} v_{8}$ and $v_{6} v_{8}$ are edges, then in Observation 2, taking $V^{\prime}=V_{8} \cup\{v\}$, we have $n^{\prime}=n-9, m^{\prime} \leq n-10$ and $\left|T^{\prime}\right| \leq(2 n-19) / 4$. Thus, $T=T^{\prime} \cup\left\{v, v_{4}, v_{5}, v_{6}\right\}$ is a transversal of $H_{G}$ of size at most $(2 n-3) / 4$, contradicting Observation 1 . Hence either $v_{1} v_{8}$ or $v_{6} v_{8}$ is an edge (but not both). 
Suppose $v_{1} v_{8}$ is an edge. Let $N\left(v_{6}\right)=\left\{v_{5}, v_{7}, v_{9}\right\}$. Then, $v_{5} v_{9}$ or $v_{7} v_{9}$ is an edge. If $v_{4} v_{9}$ is an edge, then in Observation 2, taking $V^{\prime}=V_{7} \cup\left\{v, v_{9}\right\}$, we have $n^{\prime}=n-9$, $m^{\prime} \leq n-10$ and $\left|T^{\prime}\right| \leq(2 n-19) / 4$. If $v_{8} v_{9}$ is an edge, then in Observation 2, taking $V^{\prime}=\left(V_{9} \backslash\left\{v_{4}\right\}\right) \cup\{v\}$, we have $n^{\prime}=n-9, m^{\prime} \leq n-10$ and $\left|T^{\prime}\right| \leq(2 n-19) / 4$. If $v_{9}$ is adjacent to vertex $v_{i}$, where $i \geq 10$, then taking $V^{\prime}=V_{7} \cup\left\{v, v_{9}\right\}$, we have $n^{\prime}=n-8$, $m^{\prime} \leq n-11$ and $\left|T^{\prime}\right| \leq(2 n-19) / 4$. In all three cases, $T=T^{\prime} \cup\left\{v, v_{1}, v_{6}, v_{9}\right\}$ is a transversal of $H_{G}$ of size at most $(2 n-3) / 4$, contradicting Observation 1 . Hence we must have that $d\left(v_{9}\right)=3$ and $N\left(v_{9}\right)=\left\{v_{5}, v_{6}, v_{7}\right\}$. But then in Observation 2, taking $V^{\prime}=V_{7} \cup\left\{v, v_{9}\right\}$, we have $n^{\prime}=n-9, m^{\prime} \leq n-10$ and $\left|T^{\prime}\right| \leq(2 n-19) / 4$. Thus, $T=T^{\prime} \cup\left\{v, v_{4}, v_{7}, v_{9}\right\}$ is a transversal of $H_{G}$ of size at most $(2 n-3) / 4$, contradicting Observation 1 . Hence, $v_{1} v_{8}$ is not an edge, implying that $v_{6} v_{8}$ is an edge. We may assume that $d\left(v_{1}\right)=3$ for otherwise if $v_{1}$ and $v_{7}$ have a common neighbor (not adjacent with $v_{6}$ ), then as shown earlier we reach a contradiction.

If $v_{4} v_{8}$ is not an edge, then in Observation 2, taking $V^{\prime}=V_{8} \cup\{v\}$, we have $n^{\prime}=n-9$, $m^{\prime} \leq n-10$ and $\left|T^{\prime}\right| \leq(2 n-19) / 4$. Thus, $T=T^{\prime} \cup\left\{v, v_{4}, v_{6}, v_{8}\right\}$ is a transversal of $H_{G}$ of size at most $(2 n-3) / 4$, contradicting Observation 1 . Hence, $v_{4} v_{8}$ is an edge and $d\left(v_{6}\right)=3$. But then $G=F_{10}$.

Case 1.2.2 $v_{6}$ has no common neighbor with $v_{1}$ or $v_{4}$. If $d\left(v_{1}\right)=4$ or if $d\left(v_{6}\right)=4$, then in Observation 2, taking $V^{\prime}=V_{3} \cup\left\{v, v_{6}\right\}$, we have $n^{\prime}=n-5, m^{\prime} \leq n-10$ and $\left|T^{\prime}\right| \leq(2 n-15) / 4$. Thus, $T=T^{\prime} \cup\left\{v, v_{1}, v_{6}\right\}$ is a transversal of $H_{G}$ of size at most $(2 n-3) / 4$, contradicting Observation 1. Hence, $d\left(v_{1}\right)=d\left(v_{6}\right)=3$. Similarly, $d\left(v_{4}\right)=3$. Thus by the claw-freeness of $G, v$ is the only common neighbor of $v_{1}$ and $v_{4}$. It follows that for $i \geq 6$, the vertex $v_{i}$ is at distance at least 3 from at least one vertex in $\left\{v, v_{2}, v_{3}\right\}$, and so, by Observation $5, d\left(v_{i}\right)=3$.

Suppose that $d\left(v_{5}\right)=4$. Let $N\left(v_{5}\right)=\left\{v_{2}, v_{3}, v_{6}, v_{7}\right\}$. Then, $v_{6} v_{7}$ is an edge. Let $N\left(v_{6}\right)=\left\{v_{5}, v_{7}, v_{8}\right\}$. Then, $v_{1} v_{8}$ and $v_{4} v_{8}$ are not edges. Suppose $v_{7} v_{8}$ is an edge, i.e., if $N\left(v_{7}\right)=\left\{v_{5}, v_{6}, v_{8}\right\}$. Since $G$ is claw-free, and $d\left(v_{1}\right)=d\left(v_{8}\right)=3, v_{1}$ and $v_{8}$ have no common neighbor. Thus in Observation 2, taking $V^{\prime}=\left(V_{8} \backslash\left\{v_{4}\right\}\right) \cup\{v\}$, we have $n^{\prime}=n-8, m^{\prime} \leq n-11$ and $\left|T^{\prime}\right| \leq(2 n-19) / 4$. Thus, $T=T^{\prime} \cup\left\{v, v_{1}, v_{6}, v_{8}\right\}$ is a transversal of $H_{G}$ of size at most $(2 n-3) / 4$, contradicting Observation 1 . Hence, $v_{7} v_{8}$ is not an edge. In Observation 2, taking $V^{\prime}=\left(V_{6} \backslash\left\{v_{4}\right\}\right) \cup\left\{v, v_{8}\right\}$, we have $n^{\prime}=n-7$, $m^{\prime} \leq n-11$ and $\left|T^{\prime}\right| \leq(2 n-19) / 4$. Thus, $T=T^{\prime} \cup\left\{v, v_{1}, v_{6}, v_{8}\right\}$ is a transversal of $H_{G}$ of size at most $(2 n-3) / 4$, contradicting Observation 1 . Hence, $d\left(v_{5}\right)=3$, i.e., $N\left(v_{5}\right)=\left\{v_{2}, v_{3}, v_{6}\right\}$. Let $N\left(v_{6}\right)=\left\{v_{5}, v_{7}, v_{8}\right\}$. Then, $v_{7} v_{8}$ is an edge, and there is no edge joining $\left\{v_{1}, v_{4}\right\}$ and $\left\{v_{7}, v_{8}\right\}$.

Suppose that a vertex in $\left\{v_{1}, v_{4}\right\}$ has a common neighbor with a vertex in $\left\{v_{7}, v_{8}\right\}$. We may assume that $v_{1}$ and $v_{7}$ have a common neighbor, say $v_{10}$. By the claw-freeness of $G, N\left(v_{10}\right)=\left\{v_{1}, v_{7}, v_{8}\right\}$. Thus in Observation 2, taking $V^{\prime}=\left(V_{10} \backslash\left\{v_{4}\right\}\right) \cup\{v\}$, we have $n^{\prime}=n-9, m^{\prime} \leq n-10$ and $\left|T^{\prime}\right| \leq(2 n-19) / 4$. Thus, $T=T^{\prime} \cup\left\{v, v_{1}, v_{6}, v_{7}\right\}$ is a transversal of $H_{G}$ of size at most $(2 n-3) / 4$, contradicting Observation 1 . Hence no vertex in $\left\{v_{1}, v_{4}\right\}$ has a common neighbor with a vertex in $\left\{v_{7}, v_{8}\right\}$.

Let $N\left(v_{7}\right)=\left\{v_{6}, v_{8}, v_{9}\right\}$. If $v_{8} v_{9}$ is an edge, then in Observation 2, taking $V^{\prime}=$ $\left(V_{8} \backslash\left\{v_{4}\right\}\right) \cup\{v\}$, we have $n^{\prime}=n-8, m^{\prime} \leq n-11$ and $\left|T^{\prime}\right| \leq(2 n-19) / 4$. Thus, 
$T=T^{\prime} \cup\left\{v, v_{1}, v_{6}, v_{7}\right\}$ is a transversal of $H_{G}$ of size at most $(2 n-3) / 4$, contradicting Observation 1 . Hence, $v_{8} v_{9}$ is not an edge. Let $N\left(v_{8}\right)=\left\{v_{6}, v_{7}, v_{10}\right\}$.

If $v_{9} v_{10}$ is an edge, then in Observation 2, taking $V^{\prime}=\left(V_{10} \backslash\left\{v_{1}, v_{4}\right\}\right) \cup\{v\}$, we have $n^{\prime}=n-10, m^{\prime}=n-12$ and $\left|T^{\prime}\right| \leq(2 n-22) / 4$. Thus, $T=T^{\prime} \cup\left\{v_{2}, v_{3}, v_{7}, v_{9}\right\}$ is a transversal of $H_{G}$ of size at most $(2 n-6) / 4$, contradicting Observation 1 . Hence, $v_{9} v_{10}$ is not an edge. Let $N\left(v_{9}\right)=\left\{v_{7}, v_{11}, v_{12}\right\}$.

Suppose $v_{10}$ is not adjacent to $v_{11}$ or $v_{12}$. Let $N\left(v_{10}\right)=\left\{v_{8}, v_{13}, v_{14}\right\}$. If there is an edge joining $\left\{v_{11}, v_{12}\right\}$ and $\left\{v_{13}, v_{14}\right\}$, say $v_{11} v_{13}$ is an edge, then in Observation 2, taking $V^{\prime}=\left(V_{11} \backslash V_{4}\right) \cup\left\{v, v_{13}\right\}$, we have $n^{\prime}=n-8, m^{\prime}=n-12$ and $\left|T^{\prime}\right| \leq(2 n-20) / 4$. Thus, $T=T^{\prime} \cup\left\{v_{5}, v_{6}, v_{11}, v_{13}\right\}$ is a transversal of $H_{G}$ of size at most $(2 n-4) / 4$, contradicting Observation 1. Hence there is no edge joining $\left\{v_{11}, v_{12}\right\}$ and $\left\{v_{13}, v_{14}\right\}$. Suppose that there is an edge joining $\left\{v_{1}, v_{4}\right\}$ and $\left\{v_{11}, v_{12}, v_{13}, v_{14}\right\}$, say $v_{1} v_{11}$. Then in Observation 2 , taking $V^{\prime}=\left(V_{9} \backslash\left\{v_{4}\right\}\right) \cup\left\{v, v_{11}\right\}$, we have $n^{\prime}=n-10, m^{\prime}=n-13$ and $\left|T^{\prime}\right| \leq(2 n-23) / 4$. Thus, $T=T^{\prime} \cup\left\{v, v_{1}, v_{6}, v_{8}, v_{11}\right\}$ is a transversal of $H_{G}$ of size at most $(2 n-3) / 4$, contradicting Observation 1. Hence there is no edge joining $\left\{v_{1}, v_{4}\right\}$ and $\left\{v_{11}, v_{12}, v_{13}, v_{14}\right\}$. Now at least one of $v_{11}$ or $v_{13}$, say $v_{11}$, has no common neighbor with $v_{1}$. Therefore in Observation 2, taking $V^{\prime}=\left(V_{8} \backslash\left\{v_{4}\right\}\right) \cup\left\{v, v_{11}\right\}$, we have $n^{\prime}=n-9, m^{\prime}=n-14$ and $\left|T^{\prime}\right| \leq(2 n-23) / 4$. Thus, $T=T^{\prime} \cup\left\{v, v_{1}, v_{6}, v_{8}, v_{11}\right\}$ is a transversal of $H_{G}$ of size at most $(2 n-3) / 4$, contradicting Observation 1. Hence, $v_{10}$ is adjacent to $v_{11}$ or $v_{12}$. Thus, by the clawfreeness of $G, N\left(v_{10}\right)=\left\{v_{8}, v_{11}, v_{12}\right\}$.

By the claw-freeness of $G, v$ is the only common neighbor of $v_{1}$ and $v_{4}$. Let $N\left(v_{1}\right)=$ $\left\{v, v_{2}, v_{13}\right\}$ and $N\left(v_{2}\right)=\left\{v, v_{3}, v_{14}\right\}$. If $v_{13} v_{14}$ is an edge, then in Observation 2, taking $V^{\prime}=\left(V_{13} \backslash\left\{v_{1}\right\}\right) \cup\{v\}$, we have $n^{\prime}=n-13, m^{\prime}=n-15$ and $\left|T^{\prime}\right| \leq(2 n-28) / 4$. Thus, $T=T^{\prime} \cup\left\{v_{3}, v_{5}, v_{9}, v_{10}, v_{11}, v_{14}\right\}$ is a transversal of $H_{G}$ of size at most $(2 n-4) / 4$, contradicting Observation 1 . Hence, $v_{13} v_{14}$ is not an edge. Let $N\left(v_{13}\right)=\left\{v_{1}, v_{15}, v_{16}\right\}$.

If $v_{14}$ is adjacent to $v_{15}$ or $v_{16}$, then $N\left(v_{14}\right)=\left\{v_{4}, v_{15}, v_{16}\right\}$ and the graph $G$ is fully described (and has order $n=17$ ). But then $\left\{v_{2}, v_{5}, v_{9}, v_{10}, v_{11}, v_{14}, v_{15}\right\}$, for example, is a TDS of $G$, and so $\gamma_{t}(G) \leq 7=(n-3) / 2$, a contradiction. Hence, $v_{14}$ is adjacent to neither $v_{15}$ nor $v_{16}$. Let $N\left(v_{14}\right)=\left\{v_{4}, v_{17}, v_{18}\right\}$. Then in Observation 2, taking $V^{\prime}=$ $\left(V_{15} \backslash\left\{v_{13}\right\}\right) \cup\{v\}$, we have $n^{\prime}=n-15, m^{\prime} \leq n-17$ and $\left|T^{\prime}\right| \leq(2 n-32) / 4$. Thus, $T=T^{\prime} \cup\left\{v_{2}, v_{5}, v_{9}, v_{10}, v_{11}, v_{14}, v_{15}\right\}$ is a transversal of $H_{G}$ of size at most $(2 n-4) / 4$, contradicting Observation 1.

Case 2. $v_{3} v_{5}$ is not an edge. Then, $v_{1} v_{5}$ is an edge since $G$ is claw-free.

Suppose that $v_{4} v_{5}$ is an edge. Suppose $d\left(v_{1}\right)=4$. Let $N\left(v_{1}\right)=\left\{v, v_{2}, v_{5}, v_{6}\right\}$. Then, $N\left(v_{5}\right)=\left\{v_{1}, v_{2}, v_{4}, v_{6}\right\}$. Thus in Observation 2, taking $V^{\prime}=\left(V_{5} \backslash\left\{v_{3}\right\}\right) \cup\{v\}$, we have $n^{\prime}=n-5, m^{\prime}=n-7$ and $\left|T^{\prime}\right| \leq(2 n-12) / 4$. Thus, $T=T^{\prime} \cup\left\{v, v_{1}\right\}$ is a transversal of $H_{G}$ of size at most $(2 n-4) / 4$, contradicting Observation 1 . Hence, $d\left(v_{1}\right)=3$. Thus in Observation 2, taking $V^{\prime}=\left(V_{5} \backslash\left\{v_{3}\right\}\right) \cup\{v\}$, we have $n^{\prime}=n-5, m^{\prime} \leq n-6$ and $\left|T^{\prime}\right| \leq(2 n-11) / 4$. Thus, $T=T^{\prime} \cup\left\{v, v_{4}\right\}$ is a transversal of $H_{G}$ of size at most $(2 n-4) / 4$, contradicting Observation 1 . Hence, $v_{4} v_{5}$ is not an edge.

Case 2.1 $d\left(v_{1}\right)=3$. If $d\left(v_{3}\right)=4$, then in Observation 2, taking $V^{\prime}=V_{3} \cup\{v\}$, we have $n^{\prime}=n-4, m^{\prime} \leq n-7$ and $\left|T^{\prime}\right| \leq(2 n-11) / 4$. Thus, $T=T^{\prime} \cup\left\{v_{2}, v_{3}\right\}$ is a transversal of $H_{G}$ of size at most $(2 n-3) / 4$, contradicting Observation 1. Hence, $d\left(v_{3}\right)=3$. Let 
$v_{6} \in N\left(v_{5}\right) \backslash\left\{v_{1}, v_{2}\right\}$.

Suppose that $v_{4}$ and $v_{5}$ have a common neighbor. We may assume that $v_{4} v_{6}$ is an edge. Then in Observation 2, taking $V^{\prime}=V_{6} \cup\{v\}$, we have $n^{\prime}=n-7, m^{\prime} \leq n-8$ and $\left|T^{\prime}\right| \leq(2 n-15) / 4$. Thus, $T=T^{\prime} \cup\left\{v, v_{4}, v_{6}\right\}$ is a transversal of $H_{G}$ of size at most $(2 n-3) / 4$, contradicting Observation 1 . Hence, $v_{4}$ and $v_{5}$ have no common neighbor. It follows that for $i \geq 6$, the vertex $v_{i}$ is at distance at least 3 from at least one of $v$ and $v_{2}$, and so, by Observation $5, d\left(v_{i}\right)=3$. In particular, $d\left(v_{6}\right)=3$.

If $v_{4}$ and $v_{6}$ have no common neighbor, then in Observation 2, taking $V^{\prime}=V_{4} \cup\left\{v, v_{6}\right\}$, we have $n^{\prime}=n-6, m^{\prime} \leq n-9$ and $\left|T^{\prime}\right| \leq(2 n-15) / 4$. Thus, $T=T^{\prime} \cup\left\{v, v_{4}, v_{6}\right\}$ is a transversal of $H_{G}$ of size at most $(2 n-3) / 4$, contradicting Observation 1. Hence, $v_{4}$ and $v_{6}$ have a common neighbor, $v_{7}$ say. Since $v_{4}$ and $v_{5}$ have no common neighbor, $v_{5} v_{7}$ is not an edge.

Let $N\left(v_{7}\right)=\left\{v_{4}, v_{6}, v_{8}\right\}$. If $v_{8}$ is adjacent to a vertex not in $\left\{v_{4}, v_{5}, v_{6}, v_{7}\right\}$, then in Observation 2, taking $V^{\prime}=V_{8} \cup\{v\}$, we have $n^{\prime}=n-9, m^{\prime} \leq n-10$ and $\left|T^{\prime}\right| \leq(2 n-19) / 4$. Thus, $T=T^{\prime} \cup\left\{v_{2}, v_{5}, v_{7}, v_{8}\right\}$ is a transversal of $H_{G}$ of size at most $(2 n-3) / 4$, contradicting Observation 1. Hence, $N\left(v_{8}\right) \subseteq\left\{v_{4}, v_{5}, v_{6}, v_{7}\right\}$.

On the one hand, if $v_{5} v_{8}$ is not an edge, then $N\left(v_{8}\right)=\left\{v_{4}, v_{6}, v_{7}\right\}$ and $d\left(v_{5}\right)=3$. But then $G=F_{9}$. On the other hand, if $v_{5} v_{8}$ is an edge, then since $v_{4}$ and $v_{5}$ have no common neighbor, $N\left(v_{8}\right)=\left\{v_{5}, v_{6}, v_{7}\right\}$. If now $d\left(v_{4}\right)=4$, then in Observation 2, taking $V^{\prime}=V_{8} \cup\{v\}$, we have $n^{\prime}=n-9, m^{\prime} \leq n-10$ and $\left|T^{\prime}\right| \leq(2 n-19) / 4$. Thus, $T=T^{\prime} \cup\left\{v_{1}, v_{4}, v_{5}, v_{7}\right\}$ is a transversal of $H_{G}$ of size at most $(2 n-3) / 4$, contradicting Observation 1. Hence, $d\left(v_{4}\right)=3$, and so $G=F_{9}$.

Case $2.2 d\left(v_{1}\right)=4$. Let $N\left(v_{1}\right)=\left\{v, v_{2}, v_{5}, v_{6}\right\}$. Then, $v_{5} v_{6}$ is an edge. If $d\left(v_{5}\right)=3$, then in Observation 2, taking $V^{\prime}=V_{2} \cup\left\{v, v_{5}\right\}$, we have $n^{\prime}=n-4, m^{\prime} \leq n-7$ and $\left|T^{\prime}\right| \leq(2 n-11) / 4$. Thus, $T=T^{\prime} \cup\left\{v, v_{1}\right\}$ is a transversal of $H_{G}$ of size at most $(2 n-3) / 4$, contradicting Observation 1. Hence, $d\left(v_{5}\right)=4$. Let $N\left(v_{5}\right)=\left\{v_{1}, v_{2}, v_{6}, v_{7}\right\}$. Then, $v_{6} v_{7}$ is an edge.

If $d\left(v_{3}\right)=3$, then in Observation 2, taking $V^{\prime}=V_{3} \cup\{v\}$, we have $n^{\prime}=n-4$, $m^{\prime} \leq n-7$ and $\left|T^{\prime}\right| \leq(2 n-11) / 4$. Thus, $T=T^{\prime} \cup\left\{v, v_{1}\right\}$ is a transversal of $H_{G}$ of size at most $(2 n-3) / 4$, contradicting Observation 1 . Hence, $d\left(v_{3}\right)=4$. Since $G$ is claw-free, $v_{3} v_{6}$ is not an edge. If $v_{3} v_{7}$ is an edge, then so too is $v_{4} v_{7}$. But then in Observation 2, taking $V^{\prime}=V_{5} \cup\left\{v, v_{7}\right\}$, we have $n^{\prime}=n-7, m^{\prime} \leq n-8$ and $\left|T^{\prime}\right| \leq(2 n-15) / 4$. Thus, $T=T^{\prime} \cup\left\{v, v_{1}, v_{4}\right\}$ is a transversal of $H_{G}$ of size at most $(2 n-3) / 4$, contradicting Observation 1 . Hence, $v_{3} v_{7}$ is not an edge. Let $N\left(v_{3}\right)=\left\{v, v_{2}, v_{4}, v_{8}\right\}$. Then, $v_{4} v_{8}$ is an edge.

If $d\left(v_{4}\right)=3$ or if $v_{4}$ is adjacent to $v_{6}$ or $v_{7}$, then in Observation 2, taking $V^{\prime}=V_{5} \cup\{v\}$, we have $n^{\prime}=n-6, m^{\prime} \leq n-9$ and $\left|T^{\prime}\right| \leq(2 n-15) / 4$. Thus, $T=T^{\prime} \cup\left\{v_{2}, v_{3}, v_{5}\right\}$ is a transversal of $H_{G}$ of size at most $(2 n-3) / 4$, contradicting Observation 1. Hence, $d\left(v_{4}\right)=4$ and neither $v_{4} v_{6}$ nor $v_{4} v_{7}$ is an edge. Let $N\left(v_{4}\right)=\left\{v, v_{3}, v_{8}, v_{9}\right\}$. Then, $v_{8} v_{9}$ is an edge. In Observation 2, taking $V^{\prime}=\left(V_{9} \backslash\left\{v_{7}\right\}\right) \cup\{v\}$, we have $n^{\prime}=n-9, m^{\prime} \leq n-10$ and $\left|T^{\prime}\right| \leq(2 n-19) / 4$. Thus, $T=T^{\prime} \cup\left\{v_{1}, v_{4}, v_{6}, v_{9}\right\}$ is a transversal of $H_{G}$ of size at most $(2 n-3) / 4$, contradicting Observation 1. This completes the proof of Claim 4 and of Observation 10. 
By Observation 10, we may assume that the subgraph induced by the neighborhood of every degree- 4 vertex is not isomorphic to $P_{4}$. This, together with our earlier assumptions, implies the following observation.

Observation 11 The subgraph induced by the neighborhood of every degree-4 vertex is isomorphic to $2 K_{2}$.

Since $G$ is claw-free, we have the following observation.

Observation 12 If $u$ and $w$ are adjacent vertices that do not have exactly one common neighbor, then $d(u)=d(w)=3$.

Proof. Suppose, to the contrary, that $d(u)=4$. If $u$ and $w$ have no common neighbor, then $N(u)$ induces a subgraph isomorphic to $K_{1} \cup C_{3}$, while if $u$ and $w$ have at least two common neighbors, then $N(u)$ induces a subgraph that contains a path $P_{3}$, contrary to assumption.

By Observation $11, G_{v}=2 K_{2}$. We may assume that $v_{1} v_{2}$ and $v_{3} v_{4}$ are edges.

Observation 13 If two vertices in $N(v)$ have a common neighbor different from $v$, then $G \in\left\{F_{2}, F_{3}, F_{7}\right\}$.

Proof. We may assume that $v_{1}$ and $v_{2}$ have a common neighbor $v_{5}$ different from $v$. By Observation $12, d\left(v_{1}\right)=d\left(v_{2}\right)=3$.

If $v_{5}$ is adjacent to $v_{3}$ or $v_{4}$, say to $v_{3}$, then in Observation 2, taking $V^{\prime}=V_{3} \cup\left\{v, v_{5}\right\}$, we have $n^{\prime}=n-5, m^{\prime}=n-6$ and $\left|T^{\prime}\right| \leq(2 n-11) / 4$. Thus, $T=T^{\prime} \cup\left\{v, v_{3}\right\}$ is a transversal of $H_{G}$ of size at most $(2 n-3) / 4$, contradicting Observation 1. Hence, $N\left(v_{5}\right) \cap N(v)=V_{2}$.

Case 1. $d\left(v_{5}\right)=3$. Let $N\left(v_{5}\right)=\left\{v_{1}, v_{2}, v_{6}\right\}$. By Observation 12, $d\left(v_{6}\right)=3$. If $N\left(v_{6}\right)=\left\{v_{3}, v_{4}, v_{5}\right\}$, then $G=F_{2}$. Hence we may assume that $v_{6}$ is not adjacent with both $v_{3}$ or $v_{4}$, say $v_{4} v_{6}$ is not an edge.

Suppose $v_{3} v_{6}$ is an edge. Let $N\left(v_{6}\right)=\left\{v_{3}, v_{5}, v_{7}\right\}$. Then, $v_{3} v_{7}$ is an edge. By Observation $12, v_{4} v_{7}$ is not an edge. Let $v_{8} \in N\left(v_{7}\right) \backslash\left\{v_{3}, v_{6}\right\}$. If $v_{4} v_{8}$ is an edge, then in Observation 2, taking $V^{\prime}=V_{8} \cup\{v\}$, we have $n^{\prime}=n-9, m^{\prime} \leq n-10$ and $\left|T^{\prime}\right| \leq(2 n-19) / 4$. If $v_{4} v_{8}$ is not an edge, then in Observation 2, taking $V^{\prime}=\left(V_{8} \backslash\left\{v_{4}\right\}\right) \cup\{v\}$, we have $n^{\prime}=n-8, m^{\prime}=n-11$ and $\left|T^{\prime}\right| \leq(2 n-19) / 4$. In both cases, $T=T^{\prime} \cup\left\{v, v_{1}, v_{7}, v_{8}\right\}$ is a transversal of $H_{G}$ of size at most $(2 n-3) / 4$, contradicting Observation 1 . Hence, $v_{3} v_{6}$ is not an edge. Thus, $v$ and $v_{6}$ have no common neighbor. Let $N\left(v_{6}\right)=\left\{v_{5}, v_{7}, v_{8}\right\}$. Then, $v_{7} v_{8} \in E$.

Case 1.1 There is an edge joining $\left\{v_{3}, v_{4}\right\}$ and $\left\{v_{7}, v_{8}\right\}$. We may assume that $v_{3} v_{7}$ is an edge.

If $v_{4} v_{7}$ is an edge, then by Observation $12, d\left(v_{3}\right)=d\left(v_{4}\right)=3$. In Observation 2, taking $V^{\prime}=V_{8} \cup\{v\}$, we have $n^{\prime}=n-9, m^{\prime} \leq n-10$ and $\left|T^{\prime}\right| \leq(2 n-19) / 4$. Thus, $T=T^{\prime} \cup\left\{v_{1}, v_{5}, v_{7}, v_{8}\right\}$ is a transversal of $H_{G}$ of size at most $(2 n-3) / 4$, contradicting Observation 1 . Hence, $v_{4} v_{7}$ is not an edge. 
If $v_{3} v_{8}$ is an edge, then by Observation $12, d\left(v_{7}\right)=d\left(v_{8}\right)=3$. Thus in Observation 2, taking $V^{\prime}=V_{8} \cup\{v\}$, we have $n^{\prime}=n-9, m^{\prime} \leq n-10$ and $\left|T^{\prime}\right| \leq(2 n-19) / 4$. Thus, $T=T^{\prime} \cup\left\{v_{1}, v_{3}, v_{4}, v_{5}\right\}$ is a transversal of $H_{G}$ of size at most $(2 n-3) / 4$, contradicting Observation 1 . Hence, $v_{3} v_{8}$ is not an edge.

Suppose that $d\left(v_{3}\right)=3$. Then, $d\left(v_{7}\right)=3$. If $v_{4} v_{8}$ is not an edge or if $d\left(v_{4}\right)=4$, then in Observation 2, taking $V^{\prime}=V_{8} \cup\{v\}$, we have $n^{\prime}=n-9, m^{\prime} \leq n-10$ and $\left|T^{\prime}\right| \leq(2 n-19) / 4$. Thus, $T=T^{\prime} \cup\left\{v, v_{4}, v_{6}, v_{8}\right\}$ is a transversal of $H_{G}$ of size at most $(2 n-3) / 4$, contradicting Observation 1. Hence, $v_{4} v_{8}$ is an edge and $d\left(v_{4}\right)=3$, implying that $d\left(v_{8}\right)=3$ and $G=F_{3}$. Hence we may assume that $d\left(v_{3}\right)=4$. Similarly, we may assume that $d\left(v_{4}\right)=4$.

Let $N\left(v_{3}\right)=\left\{v, v_{4}, v_{7}, v_{9}\right\}$. Then, $v_{7} v_{9}$ is an edge, and so $d\left(v_{7}\right)=4$. By Observation 12 , $v_{9}$ is adjacent to neither $v_{4}$ nor $v_{8}$. In Observation 2, taking $V^{\prime}=V_{7} \cup\{v\}$, we have $n^{\prime}=n-8, m^{\prime} \leq n-11$ and $\left|T^{\prime}\right| \leq(2 n-19) / 4$. Thus, $T=T^{\prime} \cup\left\{v, v_{4}, v_{6}, v_{7}\right\}$ is a transversal of $H_{G}$ of size at most $(2 n-3) / 4$, contradicting Observation 1.

Case 1.2 There is no edge joining $\left\{v_{3}, v_{4}\right\}$ and $\left\{v_{7}, v_{8}\right\}$. Then both $v_{7}$ and $v_{8}$ are at distance at least 3 from $v$, and so, by Observation $5, d\left(v_{7}\right)=d\left(v_{8}\right)=3$.

Suppose $v_{7}$ and $v_{8}$ have a common neighbor $v_{9}$, different from $v_{6}$. By Observation 12 , $d\left(v_{7}\right)=d\left(v_{8}\right)=3$. If $N\left(v_{9}\right) \cap\left\{v_{3}, v_{4}\right\}=\emptyset$, then in Observation 2, taking $V^{\prime}=\left(V_{9} \backslash\right.$ $\left.\left\{v_{3}, v_{4}\right\}\right) \cup\{v\}$, we have $n^{\prime}=n-8, m^{\prime}=n-11$ and $\left|T^{\prime}\right| \leq(2 n-19) / 4$. Thus, $T=T^{\prime} \cup\left\{v, v_{1}, v_{7}, v_{9}\right\}$ is a transversal of $H_{G}$ of size at most $(2 n-3) / 4$, contradicting Observation 1. Hence we may assume that $v_{3} v_{9}$ in an edge. But then in Observation 2, taking $V^{\prime}=\left(V_{9} \backslash\left\{v_{4}\right\}\right) \cup\{v\}$, we have $n^{\prime}=n-9, m^{\prime} \leq n-10$ and $\left|T^{\prime}\right| \leq(2 n-19) / 4$. Thus, $T=T^{\prime} \cup\left\{v, v_{1}, v_{7}, v_{9}\right\}$ is a transversal of $H_{G}$ of size at most $(2 n-3) / 4$, contradicting Observation 1 . Hence we may assume that $v_{6}$ is the only common neighbor of $v_{7}$ and $v_{8}$.

Let $N\left(v_{7}\right)=\left\{v_{6}, v_{8}, v_{9}\right\}$. Then, $v_{8} v_{9}$ is not an edge. By Observation $12, d\left(v_{9}\right)=3$. If $v_{3} v_{9}$ is an edge, then in Observation 2, taking $V^{\prime}=\left(V_{9} \backslash\left\{v_{4}, v_{8}\right\}\right) \cup\{v\}$, we have $n^{\prime}=n-8$, $m^{\prime}=n-11$ and $\left|T^{\prime}\right| \leq(2 n-19) / 4$. Thus, $T=T^{\prime} \cup\left\{v, v_{1}, v_{7}, v_{9}\right\}$ is a transversal of $H_{G}$ of size at most $(2 n-3) / 4$, contradicting Observation 1 . Hence, $v_{3} v_{9}$ is not an edge. Similarly, $v_{4} v_{9}$ is not an edge. But then in Observation 2, taking $V^{\prime}=\left(V_{9} \backslash\left\{v_{3}, v_{4}, v_{8}\right\}\right) \cup\{v\}$, we have $n^{\prime}=n-7, m^{\prime} \leq n-12$ and $\left|T^{\prime}\right| \leq(2 n-19) / 4$. Thus, $T=T^{\prime} \cup\left\{v, v_{1}, v_{7}, v_{9}\right\}$ is a transversal of $H_{G}$ of size at most $(2 n-3) / 4$, contradicting Observation 1.

Case 2. $d\left(v_{5}\right)=4$. Let $N\left(v_{5}\right)=\left\{v_{1}, v_{2}, v_{6}, v_{7}\right\}$. Then, $v_{6} v_{7}$ is an edge.

Case 2.1. There is an edge joining $\left\{v_{3}, v_{4}\right\}$ and $\left\{v_{6}, v_{7}\right\}$. We may assume that $v_{3} v_{6}$ is an edge. If $v_{4} v_{6}$ is an edge, then by Observation $12, d\left(v_{3}\right)=d\left(v_{4}\right)=3$. Thus in Observation 2, taking $V^{\prime}=V_{6} \cup\{v\}$, we have $n^{\prime}=n-7, m^{\prime} \leq n-8$ and $\left|T^{\prime}\right| \leq(2 n-15) / 4$. Thus, $T=T^{\prime} \cup\left\{v_{1}, v_{5}, v_{6}\right\}$ is a transversal of $H_{G}$ of size at most $(2 n-3) / 4$, contradicting Observation 1 . Hence, $v_{4} v_{6}$ is not an edge.

If $d\left(v_{6}\right)=4$, then in Observation 2, taking $V^{\prime}=\left(V_{6} \backslash\left\{v_{4}\right\}\right) \cup\{v\}$, we have $n^{\prime}=n-6$, $m^{\prime} \leq n-9$ and $\left|T^{\prime}\right| \leq(2 n-15) / 4$. Thus, $T=T^{\prime} \cup\left\{v_{3}, v_{5}, v_{6}\right\}$ is a transversal of $H_{G}$ of size at most $(2 n-3) / 4$, contradicting Observation 1. Hence, $d\left(v_{6}\right)=3$. Therefore, $d\left(v_{3}\right)=3$.

If $v_{4} v_{7}$ is an edge, then in Observation 2, taking $V^{\prime}=V_{7} \cup\{v\}$, we have $n^{\prime}=n-8$, $m^{\prime} \leq n-8$ and $\left|T^{\prime}\right| \leq(2 n-16) / 4$. Thus, $T=T^{\prime} \cup\left\{v, v_{4}, v_{7}\right\}$ is a transversal of $H_{G}$ of size at most $(2 n-4) / 4$, contradicting Observation 1 . Hence, $v_{4} v_{7}$ is not an edge. 
If $v_{4}$ and $v_{7}$ have a common neighbor, say $v_{8}$, then in Observation 2, taking $V^{\prime}=V_{8} \cup$ $\{v\}$, we have $n^{\prime}=n-9, m^{\prime} \leq n-10$ and $\left|T^{\prime}\right| \leq(2 n-19) / 4$. Thus, $T=T^{\prime} \cup\left\{v, v_{4}, v_{7}, v_{8}\right\}$ is a transversal of $H_{G}$ of size at most $(2 n-3) / 4$, contradicting Observation 1 . Hence, $v_{4}$ and $v_{7}$ have no common neighbor.

Let $v_{9} \in N\left(v_{4}\right) \backslash\left\{v, v_{3}\right\}$. In Observation 2, taking $V^{\prime}=V_{7} \cup\left\{v, v_{9}\right\}$, we have $n^{\prime}=n-9$, $m^{\prime} \leq n-10$ and $\left|T^{\prime}\right| \leq(2 n-19) / 4$. Thus, $T=T^{\prime} \cup\left\{v_{4}, v_{5}, v_{7}, v_{9}\right\}$ is a transversal of $H_{G}$ of size at most $(2 n-3) / 4$, contradicting Observation 1.

Case 2.2. There is no edge joining $\left\{v_{3}, v_{4}\right\}$ and $\left\{v_{6}, v_{7}\right\}$. Then both $v_{6}$ and $v_{7}$ are at distance 3 from $v$, and so, by Observation $5, d\left(v_{6}\right)=d\left(v_{7}\right)=3$. Let $N\left(v_{6}\right)=\left\{v_{5}, v_{7}, v_{8}\right\}$.

Suppose that there is a vertex that is a common neighbor of a vertex in $\left\{v_{3}, v_{4}\right\}$ and a vertex in $\left\{v_{6}, v_{7}\right\}$. We may assume that $v_{3} v_{8}$ is an edge. Suppose $v_{7} v_{8}$ is not an edge. Then, by Observation $12, d\left(v_{8}\right)=3$ and $v_{3}$ and $v_{8}$ have a common neighbor. If $v_{4} v_{8}$ is an edge, then by Observation $12, d\left(v_{3}\right)=d\left(v_{4}\right)=3$. Let $N\left(v_{7}\right)=\left\{v_{5}, v_{6}, v_{9}\right\}$. Then in Observation 2, taking $V^{\prime}=V_{9} \cup\{v\}$, we have $n^{\prime}=n-10, m^{\prime} \leq n-12$ and $\left|T^{\prime}\right| \leq(2 n-22) / 4$. Thus, $T=T^{\prime} \cup\left\{v, v_{3}, v_{7}, v_{9}\right\}$ is a transversal of $H_{G}$ of size at most $(2 n-6) / 4$, contradicting Observation 1 . Hence, $v_{4} v_{8}$ is not an edge. But then in Observation 2, taking $V^{\prime}=V_{6} \cup\left\{v, v_{8}\right\}$, we have $n^{\prime}=n-8, m^{\prime} \leq n-11$ and $\left|T^{\prime}\right| \leq(2 n-19) / 4$. Thus, $T=T^{\prime} \cup\left\{v, v_{4}, v_{6}, v_{8}\right\}$ is a transversal of $H_{G}$ of size at most $(2 n-3) / 4$, contradicting Observation 1 . Hence, $v_{7} v_{8}$ is an edge. If $v_{4} v_{8}$ is not an edge, then in Observation 2, taking $V^{\prime}=V_{8} \cup\{v\}$, we have $n^{\prime}=n-9, m^{\prime} \leq n-10$ and $\left|T^{\prime}\right| \leq(2 n-19) / 4$. Thus, $T=T^{\prime} \cup\left\{v, v_{4}, v_{6}, v_{8}\right\}$ is a transversal of $H_{G}$ of size at most $(2 n-3) / 4$, contradicting Observation 1. Hence, $v_{4} v_{8}$ is an edge, and so $G=F_{7}$.

Hence we may assume that no vertex is a common neighbor of a vertex in $\left\{v_{3}, v_{4}\right\}$ and a vertex in $\left\{v_{6}, v_{7}\right\}$, for otherwise $G=F_{7}$. Thus, $d\left(v, v_{8}\right) \geq 3$, and so, by Observation 5 , $d\left(v_{8}\right)=3$.

Suppose that $v_{3}$ or $v_{4}$, say $v_{3}$, has degree 3. Then in Observation 2, taking $V^{\prime}=$ $V_{2} \cup\left\{v, v_{3}, v_{6}\right\}$, we have $n^{\prime}=n-5, m^{\prime} \leq n-10$ and $\left|T^{\prime}\right| \leq(2 n-15) / 4$. Thus, $T=T^{\prime} \cup\left\{v, v_{3}, v_{6}\right\}$ is a transversal of $H_{G}$ of size at most $(2 n-3) / 4$, contradicting Observation 1 . Hence, $d\left(v_{3}\right)=d\left(v_{4}\right)=3$.

If $v_{3}$ and $v_{4}$ have a common neighbor different from $v$, then in Observation 2, taking $V^{\prime}=V_{4} \cup\left\{v, v_{6}\right\}$, we have $n^{\prime}=n-6, m^{\prime} \leq n-9$ and $\left|T^{\prime}\right| \leq(2 n-15) / 4$. Thus, $T=T^{\prime} \cup\left\{v, v_{3}, v_{6}\right\}$ is a transversal of $H_{G}$ of size at most $(2 n-3) / 4$, contradicting Observation 1. Hence, $v$ is the only common neighbor of $v_{3}$ and $v_{4}$. Let $N\left(v_{3}\right)=\left\{v, v_{4}, v_{9}\right\}$. By Observation 12, $d\left(v_{9}\right)=3$.

If $v_{7} v_{8}$ is an edge, then in Observation 2, taking $V^{\prime}=V_{7} \backslash\left\{v_{4}\right\}$, we have $n^{\prime}=n-6$, $m^{\prime} \leq n-9$ and $\left|T^{\prime}\right| \leq(2 n-15) / 4$. Thus, $T=T^{\prime} \cup\left\{v_{3}, v_{5}, v_{6}\right\}$ is a transversal of $H_{G}$ of size at most $(2 n-3) / 4$, contradicting Observation 1 . Hence, $v_{7} v_{8}$ is not an edge. If $v_{8} v_{9}$ is not an edge, then in Observation 2, taking $V^{\prime}=V_{3} \cup\left\{v, v_{5}, v_{6}, v_{9}\right\}$, we have $n^{\prime}=n-7$, $m^{\prime}=n-12$ and $\left|T^{\prime}\right| \leq(2 n-19) / 4$. Thus, $T=T^{\prime} \cup\left\{v_{3}, v_{5}, v_{6}, v_{9}\right\}$ is a transversal of $H_{G}$ of size at most $(2 n-3) / 4$, contradicting Observation 1 . Hence, $v_{8} v_{9}$ is an edge.

Let $v_{10}$ be the common neighbor of $v_{8}$ and $v_{9}$, and so $N\left(v_{8}\right)=\left\{v_{6}, v_{9}, v_{10}\right\}$ and $N\left(v_{9}\right)=$ $\left\{v_{3}, v_{8}, v_{10}\right\}$. Then in Observation 2, taking $V^{\prime}=\left(V_{9} \backslash\left\{v_{4}\right\}\right) \cup\{v\}$, we have $n^{\prime}=n-9$, $m^{\prime} \leq n-11$ and $\left|T^{\prime}\right| \leq(2 n-20) / 4$. Thus, $T=T^{\prime} \cup\left\{v_{3}, v_{5}, v_{7}, v_{9}\right\}$ is a transversal of 
$H_{G}$ of size at most $(2 n-4) / 4$, contradicting Observation 1 . This completes the proof of Observation 13.

By Observation 13, we may assume that no two vertices in $N(v)$ have a common neighbor different from $v$. Thus, $N\left(v_{i}\right) \cap N\left(v_{j}\right)=\{v\}$ for $1 \leq i<j \leq 4$. For $i=1,2,3,4$, let $v_{i+4}$ be the neighbor of $v_{i}$ not in $N[v]$. Thus, $\left\{v_{1} v_{5}, v_{2} v_{6}, v_{3} v_{7}, v_{4} v_{8}\right\} \subset E$.

Observation 14 There is no 4-cycle containing both $v_{1}$ and $v_{2}$ or containing both $v_{3}$ and $v_{4}$.

Proof. Suppose, to the contrary, that there is a 4-cycle containing both $v_{1}$ and $v_{2}$ or containing both $v_{3}$ and $v_{4}$. By symmetry, we may assume there is a 4-cycle containing both $v_{1}$ and $v_{2}$ and that $v_{5} v_{6}$ is an edge.

Case 1. $v_{1}$ or $v_{2}$ has degree 4 . We may assume that $d\left(v_{1}\right)=4$. Let $N\left(v_{1}\right)=$ $\left\{v, v_{2}, v_{5}, v_{9}\right\}$. Then, $v_{5} v_{9}$ is an edge. If $v_{6} v_{9}$ is an edge, then by Observation $12, d\left(v_{5}\right)=$ $d\left(v_{9}\right)=3$. Thus in Observation 2, taking $V^{\prime}=V_{2} \cup\left\{v_{5}, v_{6}, v_{9}\right\}$, we have $n^{\prime}=n-5$, $m^{\prime}=n-6$ and $\left|T^{\prime}\right| \leq(2 n-11) / 4$. Thus, $T=T^{\prime} \cup\left\{v_{2}, v_{6}\right\}$ is a transversal of $H_{G}$ of size at most $(2 n-3) / 4$, contradicting Observation 1. Hence, $v_{6} v_{9}$ is not an edge. Every neighbor of $v_{6}$, different from $v_{2}$ and $v_{5}$, is adjacent to $v_{2}$ or $v_{5}$.

Suppose that $v_{6}$ is adjacent to $v_{7}$ or $v_{8}$, say $v_{7}$. Then, $v_{5} v_{7}$ is an edge. If $v_{3}$ or $v_{4}$ or $v_{6}$ has degree 4, then in Observation 2, taking $V^{\prime}=V_{7} \cup\{v\}$, we have $n^{\prime}=n-8$, $m^{\prime} \leq n-11$ and $\left|T^{\prime}\right| \leq(2 n-19) / 4$. Thus, $T=T^{\prime} \cup\left\{v_{3}, v_{4}, v_{5}, v_{6}\right\}$ is a transversal of $H_{G}$ of size at most $(2 n-3) / 4$, contradicting Observation 1. Hence, $d\left(v_{3}\right)=d\left(v_{4}\right)=d\left(v_{6}\right)=3$. Thus in Observation 2, taking $V^{\prime}=\left(V_{7} \backslash\left\{v_{4}\right\}\right) \cup\{v\}$, we have $n^{\prime}=n-7, m^{\prime}=n-9$ and $\left|T^{\prime}\right| \leq(2 n-16) / 4$. Thus, $T=T^{\prime} \cup\left\{v, v_{1}, v_{5}\right\}$ is a transversal of $H_{G}$ of size at most $(2 n-4) / 4$, contradicting Observation 1 . Hence, $v_{6}$ is adjacent to neither $v_{7}$ nor $v_{8}$. By the claw-freeness of $G, v_{5}$ is also adjacent to neither $v_{7}$ nor $v_{8}$.

If one of $v_{3}$ or $v_{4}$, say $v_{3}$, has degree 4 or if $d\left(v_{6}\right)=4$, then in Observation 2, taking $V^{\prime}=V_{3} \cup\left\{v_{5}, v_{6}\right\}$, we have $n^{\prime}=n-5, m^{\prime} \leq n-10$ and $\left|T^{\prime}\right| \leq(2 n-15) / 4$. Thus, $T=T^{\prime} \cup\left\{v_{3}, v_{5}, v_{6}\right\}$ is a transversal of $H_{G}$ of size at most $(2 n-3) / 4$, contradicting Observation 1. Hence, $d\left(v_{3}\right)=d\left(v_{4}\right)=d\left(v_{6}\right)=3$. Thus, by Observation $12, d\left(v_{7}\right)=$ $d\left(v_{8}\right)=3$. Let $N\left(v_{6}\right)=\left\{v_{2}, v_{5}, v_{10}\right\}$.

If $v_{7} v_{8}$ is an edge, then in Observation 2, taking $V^{\prime}=V_{8} \cup\{v\}$, we have $n^{\prime}=n-9$, $m^{\prime} \leq n-11$ and $\left|T^{\prime}\right| \leq(2 n-20) / 4$. Thus, $T=T^{\prime} \cup\left\{v_{3}, v_{5}, v_{6}, v_{7}\right\}$ is a transversal of $H_{G}$ of size at most $(2 n-3) / 4$, contradicting Observation 1 . Hence, $v_{7} v_{8}$ is not an edge.

If $v_{7}$ or $v_{8}$, say $v_{7}$, is adjacent to neither $v_{9}$ nor $v_{10}$, then in Observation 2, taking $V^{\prime}=\left(V_{7} \backslash\left\{v_{4}\right\}\right) \cup\{v\}$, we have $n^{\prime}=n-7, m^{\prime}=n-12$ and $\left|T^{\prime}\right| \leq(2 n-19) / 4$. Thus, $T=T^{\prime} \cup\left\{v_{3}, v_{5}, v_{6}, v_{7}\right\}$ is a transversal of $H_{G}$ of size at most $(2 n-3) / 4$, contradicting Observation 1. Hence each of $v_{7}$ and $v_{8}$ is adjacent to at least one of $v_{9}$ and $v_{10}$. By the claw-freeness of $G$, each of $v_{7}$ and $v_{8}$ is adjacent to at most one of $v_{9}$ and $v_{10}$. Hence we may assume that $N\left(v_{7}\right)=\left\{v_{3}, v_{9}, v_{11}\right\}$ and $N\left(v_{8}\right)=\left\{v_{4}, v_{10}, v_{12}\right\}$. Thus, $v_{9} v_{11}$ is an edge and $v_{10} v_{12}$ is an edge. In Observation 2, taking $V^{\prime}=V_{10} \cup\{v\}$, we have $n^{\prime}=n-11$, $m^{\prime} \leq n-13$ and $\left|T^{\prime}\right| \leq(2 n-24) / 4$. Thus, $T=T^{\prime} \cup\left\{v, v_{1}, v_{8}, v_{9}, v_{10}\right\}$ is a transversal of $H_{G}$ of size at most $(2 n-4) / 4$, contradicting Observation 1. 
Case 2. $d\left(v_{1}\right)=d\left(v_{2}\right)=3$. Thus by Observation $12, d\left(v_{5}\right)=d\left(v_{6}\right)=3$.

If $v_{7}$ or $v_{8}$, say $v_{7}$, is the common neighbor of $v_{5}$ and $v_{6}$, then in Observation 2, taking $V^{\prime}=\left(V_{7} \backslash\left\{v_{4}\right\}\right) \cup\{v\}$, we have $n^{\prime}=n-7, m^{\prime} \leq n-8$ and $\left|T^{\prime}\right| \leq(2 n-15) / 4$. Thus, $T=T^{\prime} \cup\left\{v, v_{3}, v_{7}\right\}$ is a transversal of $H_{G}$ of size at most $(2 n-3) / 4$, contradicting Observation 1 . Hence neither $v_{7}$ nor $v_{8}$ is the common neighbor of $v_{5}$ and $v_{6}$. Let $v_{9}$ be the common neighbor of $v_{5}$ and $v_{6}$.

Suppose that $v_{9}$ has a common neighbor with $v_{3}$ or $v_{4}$. We may assume that $v_{7} v_{9}$ is an edge. Then in Observation 2, taking $V^{\prime}=V_{7} \cup\left\{v, v_{9}\right\}$, we have $n^{\prime}=n-9, m^{\prime} \leq n-10$ and $\left|T^{\prime}\right| \leq(2 n-19) / 4$. Thus, $T=T^{\prime} \cup\left\{v, v_{4}, v_{7}, v_{9}\right\}$ is a transversal of $H_{G}$ of size at most $(2 n-3) / 4$, contradicting Observation 1. Hence, $v_{9}$ has no common neighbor with $v_{3}$ or $v_{4}$. In particular, $v_{9}$ is adjacent to neither $v_{7}$ nor $v_{8}$. Thus, $d\left(v, v_{9}\right)=3$, and so, by Observation $5, d\left(v_{9}\right)=3$. Let $N\left(v_{9}\right)=\left\{v_{5}, v_{6}, v_{10}\right\}$. Then in Observation 2, taking $V^{\prime}=V_{3} \cup\left\{v, v_{5}, v_{6}, v_{9}, v_{10}\right\}$, we have $n^{\prime}=n-8, m^{\prime} \leq n-11$ and $\left|T^{\prime}\right| \leq(2 n-19) / 4$. Thus, $T=T^{\prime} \cup\left\{v, v_{3}, v_{9}, v_{10}\right\}$ is a transversal of $H_{G}$ of size at most $(2 n-3) / 4$, contradicting Observation 1.

Since both Case 1 and Case 2 produce a contradiction, we conclude that $v_{5} v_{6}$ is not an edge, i.e., there is no 4 -cycle containing both $v_{1}$ and $v_{2}$ or containing both $v_{3}$ and $v_{4}$.

Observation $15\left\{v_{5}, v_{6}, v_{7}, v_{8}\right\}$ is an independent set.

Proof. Assume, to the contrary, that $\left\{v_{5}, v_{6}, v_{7}, v_{8}\right\}$ is not an independent set. Then, by Observation 14, there is an edge joining a vertex in $\left\{v_{5}, v_{6}\right\}$ with a vertex in $\left\{v_{7}, v_{8}\right\}$. We may assume that $v_{6} v_{7} \in E$. We show first that $v_{6}$ and $v_{7}$ have a common neighbor.

Claim $5 v_{6}$ and $v_{7}$ have a common neighbor.

Proof. Suppose, to the contrary, that $v_{6}$ and $v_{7}$ have no common neighbor. Then, by Observation 12, $d\left(v_{6}\right)=d\left(v_{7}\right)=3$. Let $N\left(v_{6}\right)=\left\{v_{2}, v_{7}, v_{9}\right\}$ and let $N\left(v_{7}\right)=\left\{v_{3}, v_{6}, v_{10}\right\}$. Then, $v_{2} v_{9}$ and $v_{3} v_{10}$ are edges, and $d\left(v_{2}\right)=d\left(v_{3}\right)=4$. By Observation 14, $v_{5} v_{9}$ is not an edge and $v_{8} v_{10}$ is not an edge.

If $v_{5} v_{10}$ is an edge, then in Observation 2, taking $V^{\prime}=\left(V_{7} \backslash\left\{v_{4}\right\}\right) \cup\left\{v, v_{10}\right\}$, we have $n^{\prime}=n-8, m^{\prime} \leq n-11$ and $\left|T^{\prime}\right| \leq(2 n-19) / 4$. Thus, $T=T^{\prime} \cup\left\{v, v_{2}, v_{5}, v_{10}\right\}$ is a transversal of $H_{G}$ of size at most $(2 n-3) / 4$, contradicting Observation 1. Hence, $v_{5} v_{10}$ is not an edge. Similarly, $v_{8} v_{9}$ is not an edge.

Suppose $v_{9} v_{10}$ is an edge. If $d\left(v_{1}\right)=4$ or if $d\left(v_{9}\right)=4$ or if $d\left(v_{10}\right)=4$, then in Observation 2, taking $V^{\prime}=V_{3} \cup\left\{v, v_{6}, v_{7}, v_{9}, v_{10}\right\}$, we have $n^{\prime}=n-8, m^{\prime} \leq n-11$ and $\left|T^{\prime}\right| \leq(2 n-19) / 4$. Thus, $T=T^{\prime} \cup\left\{v, v_{1}, v_{9}, v_{10}\right\}$ is a transversal of $H_{G}$ of size at most $(2 n-3) / 4$, contradicting Observation 1. Hence, $d\left(v_{1}\right)=d\left(v_{9}\right)=d\left(v_{10}\right)=3$. Similarly, $d\left(v_{4}\right)=3$. But then in Observation 2, taking $V^{\prime}=\left(V_{10} \backslash\left\{v_{5}, v_{8}\right\}\right) \cup\{v\}$, we have $n^{\prime}=n-9, m^{\prime} \leq n-11$ and $\left|T^{\prime}\right| \leq(2 n-20) / 4$. Thus, $T=T^{\prime} \cup\left\{v_{1}, v_{2}, v_{3}, v_{4}\right\}$ is a transversal of $H_{G}$ of size at most $(2 n-4) / 4$, contradicting Observation 1 . Hence, $v_{9} v_{10}$ is not an edge.

Suppose $v_{5} v_{8}$ is an edge. Suppose $v_{5}$ and $v_{8}$ have a common neighbor, say $v_{11}$. Then, in Observation 2, taking $V^{\prime}=\left(V_{7} \backslash\left\{v_{5}\right\}\right) \cup\left\{v, v_{11}\right\}$, we have $n^{\prime}=n-8, m^{\prime} \leq n-11$ 
and $\left|T^{\prime}\right| \leq(2 n-19) / 4$. Thus, $T=T^{\prime} \cup\left\{v, v_{2}, v_{3}, v_{11}\right\}$ is a transversal of $H_{G}$ of size at most $(2 n-3) / 4$, contradicting Observation 1. Hence, $v_{5}$ and $v_{8}$ have no common neighbor. Thus by Observation $12, d\left(v_{5}\right)=d\left(v_{8}\right)=3$. Let $N\left(v_{5}\right)=\left\{v_{1}, v_{8}, v_{11}\right\}$ and let $N\left(v_{8}\right)=\left\{v_{4}, v_{5}, v_{12}\right\}$. Thus, $v_{1} v_{11}$ and $v_{4} v_{12}$ are edges, and $d\left(v_{1}\right)=d\left(v_{4}\right)=4$. A similar argument to the one that show that $v_{9} v_{10}$ is not an edge, shows that $v_{11} v_{12}$ is not an edge.

By Observation 14, we know that neither $v_{9} v_{11}$ nor $v_{10} v_{12}$ is an edge. Suppose that $v_{9} v_{12}$ or $v_{10} v_{11}$ is an edge. By symmetry, we may assume $v_{9} v_{12}$ is an edge. In Observation 2, taking $V^{\prime}=V_{9} \cup\left\{v, v_{12}\right\}$, we have $n^{\prime}=n-11, m^{\prime} \leq n-13$ and $\left|T^{\prime}\right| \leq(2 n-24) / 4$. Thus, $T=T^{\prime} \cup\left\{v, v_{1}, v_{3}, v_{9}, v_{12}\right\}$ is a transversal of $H_{G}$ of size at most $(2 n-4) / 4$, contradicting Observation 1 . Hence, neither $v_{9} v_{12}$ nor $v_{10} v_{11}$ is an edge. Thus, $\left\{v_{9}, v_{10}, v_{11}, v_{12}\right\}$ is an independent set.

Let $v_{13} \in N\left(v_{12}\right)$. Since $\left\{v_{9}, v_{10}, v_{11}, v_{12}\right\}$ is an independent set, $v_{13}$ is adjacent to at most two vertices in $\left\{v_{9}, v_{10}, v_{11}, v_{12}\right\}$. Thus, $v_{13}$ has at least one neighbor not in the set $\left\{v_{9}, v_{10}, v_{11}, v_{12}\right\}$. Therefore in Observation 2, taking $V^{\prime}=V_{8} \cup\left\{v, v_{11}, v_{12}, v_{13}\right\}$, we have $n^{\prime}=n-12, m^{\prime} \leq n-15$ and $\left|T^{\prime}\right| \leq(2 n-27) / 4$. Thus, $T=T^{\prime} \cup\left\{v_{1}, v_{6}, v_{7}, v_{11}, v_{12}, v_{13}\right\}$ is a transversal of $H_{G}$ of size at most $(2 n-3) / 4$, contradicting Observation 1 . This completes the proof of Claim 5.

By Claim 5, $v_{6}$ and $v_{7}$ have a common neighbor, say $v_{9}$. By Observation $14, v_{9}$ is adjacent to neither $v_{1}$ nor $v_{4}$. Thus, $d\left(v, v_{9}\right)=3$, and so, by Observation $4, d\left(v_{9}\right)=3$.

Suppose that $d\left(v_{2}\right)=d\left(v_{3}\right)=3$. Then, by Observation $12, d\left(v_{6}\right)=d\left(v_{7}\right)=3$. Suppose that $v_{9}$ is adjacent to $v_{5}$ or $v_{8}$, say $v_{5}$. Then in Observation 2, taking $V^{\prime}=V_{7} \cup\left\{v, v_{9}\right\}$, we have $n^{\prime}=n-9, m^{\prime} \leq n-10$ and $\left|T^{\prime}\right| \leq(2 n-19) / 4$. Thus, $T=T^{\prime} \cup\left\{v, v_{4}, v_{5}, v_{9}\right\}$ is a transversal of $H_{G}$ of size at most $(2 n-3) / 4$, contradicting Observation 1. Hence, neither $v_{5} v_{9}$ nor $v_{8} v_{9}$ is an edge. Let $N\left(v_{9}\right)=\left\{v_{6}, v_{7}, v_{10}\right\}$. In Observation 2, taking $V^{\prime}=V_{3} \cup\left\{v, v_{6}, v_{7}, v_{9}, v_{10}\right\}$, we have $n^{\prime}=n-8, m^{\prime} \leq n-11$ and $\left|T^{\prime}\right| \leq(2 n-19) / 4$. Thus, $T=T^{\prime} \cup\left\{v, v_{1}, v_{9}, v_{10}\right\}$ is a transversal of $H_{G}$ of size at most $(2 n-3) / 4$, contradicting Observation 1 . Hence at least one of $v_{2}$ and $v_{3}$ has degree 4 .

If $v_{2} v_{9}$ is an edge, then by Observation $12, d\left(v_{6}\right)=d\left(v_{9}\right)=3$, and so in Observation 2, taking $V^{\prime}=\left(V_{7} \backslash\left\{v_{4}\right\}\right) \cup\left\{v, v_{9}\right\}$, we have $n^{\prime}=n-8, m^{\prime} \leq n-11$ and $\left|T^{\prime}\right| \leq(2 n-19) / 4$. Thus, $T=T^{\prime} \cup\left\{v_{1}, v_{3}, v_{5}, v_{7}\right\}$ is a transversal of $H_{G}$ of size at most $(2 n-3) / 4$, contradicting Observation 1. Hence, $v_{2} v_{9}$ is not an edge. Similarly, $v_{3} v_{9}$ is not an edge. Thus, if $d\left(v_{2}\right)=4$, then $v_{2}$ and $v_{6}$ have a common neighbor which is different from $v_{9}$, while if $d\left(v_{3}\right)=4$, then $v_{3}$ and $v_{7}$ have a common neighbor which is different from $v_{9}$. In particular, $d\left(v_{6}\right)=4$ or $d\left(v_{7}\right)=4$.

Suppose $v_{9}$ is adjacent to $v_{5}$ or $v_{8}$, say $v_{5}$. By Observation $12, d\left(v_{5}\right)=3$. Hence, $d\left(v_{1}\right)=4$ and $v_{1}$ and $v_{5}$ have a common neighbor. In Observation 2 , taking $V^{\prime}=$ $\left(V_{7} \backslash\left\{v_{4}\right\}\right) \cup\left\{v, v_{9}\right\}$, we have $n^{\prime}=n-8, m^{\prime} \leq n-11$ and $\left|T^{\prime}\right| \leq(2 n-19) / 4$. Thus, $T=T^{\prime} \cup\left\{v, v_{1}, v_{6}, v_{7}\right\}$ is a transversal of $H_{G}$ of size at most $(2 n-3) / 4$, contradicting Observation 1 . Hence, $v_{9}$ is adjacent to neither $v_{5}$ nor $v_{8}$. Let $N\left(v_{9}\right)=\left\{v_{6}, v_{7}, v_{10}\right\}$. By Observation $12, d\left(v_{10}\right)=3$.

If $v_{10}$ is adjacent to $v_{2}$ or $v_{3}$, say $v_{2}$, then $v_{6} v_{10}$ is an edge, and so $N\left(v_{6}\right)$ induces a subgraph that contains a $P_{4}$, contradicting Observation 11. Hence, $v_{10}$ is adjacent to neither $v_{2}$ nor $v_{3}$. If $v_{10}$ is adjacent to $v_{1}$ or $v_{4}$, say $v_{1}$, then $v_{5} v_{10}$ is an edge. But then 
in Observation 2, taking $V^{\prime}=V_{3} \cup\left\{v, v_{6}, v_{7}, v_{9}, v_{10}\right\}$, we have $n^{\prime}=n-8, m^{\prime} \leq n-11$ and $\left|T^{\prime}\right| \leq(2 n-19) / 4$. Thus, $T=T^{\prime} \cup\left\{v, v_{1}, v_{6}, v_{7}\right\}$ is a transversal of $H_{G}$ of size at most $(2 n-3) / 4$, contradicting Observation 1 . Hence, $v_{10}$ is adjacent to no vertex in $N(v)$.

If $v_{10}$ is adjacent to $v_{5}$ or $v_{8}$, say $v_{5}$, then $v_{5}$ and $v_{10}$ have a common neighbor. In Observation 2, taking $V^{\prime}=\left(V_{10} \backslash\left\{v_{4}\right\}\right) \cup\{v\}$, we have $n^{\prime}=n-10, m^{\prime} \leq n-14$ and $\left|T^{\prime}\right| \leq(2 n-24) / 4$. Thus, $T=T^{\prime} \cup\left\{v_{1}, v_{5}, v_{6}, v_{7}, v_{8}\right\}$ is a transversal of $H_{G}$ of size at most $(2 n-4) / 4$, contradicting Observation 1 . Hence, $v_{10}$ is adjacent to neither $v_{5}$ nor $v_{8}$. Let $N\left(v_{10}\right)=\left\{v_{9}, v_{11}, v_{12}\right\}$. Then, $v_{11} v_{12}$ is an edge.

Suppose there is an edge joining a vertex in $\left\{v_{2}, v_{3}\right\}$ with a vertex in $\left\{v_{11}, v_{12}\right\}$. We may assume $v_{2} v_{11}$ is an edge. Then $v_{6} v_{11}$ is an edge and in Observation 2, taking $V^{\prime}=$ $\left(V_{11} \backslash\left\{v_{1}, v_{4}, v_{5}, v_{8}\right\}\right) \cup\{v\}$, we have $n^{\prime}=n-8, m^{\prime} \leq n-11$ and $\left|T^{\prime}\right| \leq(2 n-19) / 4$. Thus, $T=T^{\prime} \cup\left\{v, v_{3}, v_{10}, v_{11}\right\}$ is a transversal of $H_{G}$ of size at most $(2 n-3) / 4$, contradicting Observation 1. Hence, there is no edge joining $\left\{v_{2}, v_{3}\right\}$ and $\left\{v_{11}, v_{12}\right\}$.

Suppose there is an edge joining a vertex in $\left\{v_{1}, v_{4}\right\}$ with a vertex in $\left\{v_{11}, v_{12}\right\}$. We may assume $v_{1} v_{11}$ is an edge. Then, $v_{5} v_{11}$ is an edge. But then in Observation 2, taking $V^{\prime}=\left(V_{11} \backslash\left\{v_{4}, v_{5}\right\}\right) \cup\{v\}$, we have $n^{\prime}=n-10, m^{\prime} \leq n-13$ and $\left|T^{\prime}\right| \leq(2 n-23) / 4$. Thus, $T=T^{\prime} \cup\left\{v_{1}, v_{6}, v_{7}, v_{8}, v_{11}\right\}$ is a transversal of $H_{G}$ of size at most $(2 n-3) / 4$, contradicting Observation 1. Hence, neither $v_{11}$ nor $v_{12}$ is adjacent to a vertex in $N(v)$. Thus, by Observation $4, d\left(v_{11}\right)=d\left(v_{12}\right)=3$.

Suppose there is an edge joining a vertex in $\left\{v_{5}, v_{8}\right\}$ with a vertex in $\left\{v_{11}, v_{12}\right\}$. We may assume $v_{5} v_{11}$ is an edge. By Observation 12, $d\left(v_{5}\right)=3$. Hence, $d\left(v_{1}\right)=4$ and $v_{1}$ and $v_{5}$ have a common neighbor. If $v_{8} v_{12}$ is not an edge, then in Observation 2, taking $V^{\prime}=\left(V_{11} \backslash\left\{v_{4}\right\}\right) \cup\{v\}$, we have $n^{\prime}=n-11, m^{\prime} \leq n-16$ and $\left|T^{\prime}\right| \leq(2 n-27) / 4$. Thus, $T=T^{\prime} \cup\left\{v_{1}, v_{5}, v_{6}, v_{7}, v_{8}, v_{11}\right\}$ is a transversal of $H_{G}$ of size at most $(2 n-3) / 4$, contradicting Observation 1. Hence, $v_{8} v_{12}$ is an edge. By Observation $12, d\left(v_{8}\right)=3$. Hence, $d\left(v_{4}\right)=4$ and $v_{4}$ and $v_{8}$ have a common neighbor. In Observation 2, taking $V^{\prime}=\left(V_{11} \backslash\left\{v_{1}, v_{5}, v_{8}\right\}\right) \cup\{v\}$, we have $n^{\prime}=n-9, m^{\prime} \leq n-14$ and $\left|T^{\prime}\right| \leq(2 n-23) / 4$. Thus, $T=\left\{v, v_{4}, v_{6}, v_{7}, v_{11}\right\}$ is a transversal of $H_{G}$ of size at most $(2 n-3) / 4$, contradicting Observation 1. Hence, there is no edge joining $\left\{v_{5}, v_{8}\right\}$ and $\left\{v_{11}, v_{12}\right\}$.

Suppose $v_{5} v_{8}$ is an edge. As in Claim 5, we must have that $v_{5}$ and $v_{8}$ have a common neighbor. Further, as shown with the $v_{6}$ and $v_{7}$, at least one of $v_{5}$ and $v_{8}$ has degree 4 . Hence, in Observation 2, taking $V^{\prime}=V_{9} \cup\left\{v, v_{11}\right\}$, we have $n^{\prime}=n-11, m^{\prime} \leq n-16$ and $\left|T^{\prime}\right| \leq(2 n-27) / 4$. Thus, $T=T^{\prime} \cup\left\{v_{1}, v_{5}, v_{6}, v_{7}, v_{8}, v_{11}\right\}$ is a transversal of $H_{G}$ of size at most $(2 n-3) / 4$, contradicting Observation 1 . Hence, $v_{5} v_{8}$ is not an edge.

If $v_{5}$ and $v_{8}$ have no common neighbor, then in Observation 2, taking $V^{\prime}=\left(V_{9} \backslash\right.$ $\left.\left\{v_{4}\right\}\right) \cup\left\{v, v_{11}\right\}$, we have $n^{\prime}=n-10, m^{\prime} \leq n-17$ and $\left|T^{\prime}\right| \leq(2 n-27) / 4$. Thus, $T=$ $T^{\prime} \cup\left\{v_{1}, v_{5}, v_{6}, v_{7}, v_{8}, v_{11}\right\}$ is a transversal of $H_{G}$ of size at most $(2 n-3) / 4$, contradicting Observation 1. Hence, $v_{5}$ and $v_{8}$ have a common neighbor. Such a common neighbor is at distance at least 3 from both $v_{6}$ and $v_{7}$, and so, by Observation 5 , has degree 3 . Hence, $v_{5}$ and $v_{8}$ have two common neighbor (of degree 3 ), say $v_{13}$ and $v_{14}$. But then in Observation 2, taking $V^{\prime}=V_{8} \cup\left\{v_{13}, v_{14}\right\}$, we have $n^{\prime}=n-11, m^{\prime} \leq n-13$ and $\left|T^{\prime}\right| \leq$ $(2 n-24) / 4$. Thus, $T=T^{\prime} \cup\left\{v_{3}, v_{5}, v_{6}, v_{7}, v_{13}\right\}$ is a transversal of $H_{G}$ of size at most $(2 n-$ 4)/4, contradicting Observation 1. This completes the proof of Observation 15. 
By Observation 15, $\left\{v_{5}, v_{6}, v_{7}, v_{8}\right\}$ is an independent set.

Observation 16 If every neighbor of $v$ has degree 3 , then $G \in\left\{F_{11}, F_{12}\right\}$.

Proof. By Observation 12, we have that $d\left(v_{i}\right)=3$ for $i \in\{5,6,7,8\}$. By Observation 4, it follows that $v$ is therefore the only degree- 4 vertex in $G$. Let $N\left(v_{5}\right)=\left\{v_{1}, v_{9}, v_{10}\right\}$. Then, $v_{9} v_{10} \in E$.

Suppose first that a vertex in $\left\{v_{5}, v_{6}\right\}$ and a vertex in $\left\{v_{7}, v_{8}\right\}$ have a common neighbor. We may assume that $v_{5}$ and $v_{7}$ have a common neighbor. Thus, $N\left(v_{7}\right)=\left\{v_{3}, v_{9}, v_{10}\right\}$. Suppose that $v_{6}$ and $v_{8}$ have no common neighbor. Let $N\left(v_{6}\right)=\left\{v_{2}, v_{11}, v_{12}\right\}$ and $N\left(v_{8}\right)=$ $\left\{v_{4}, v_{13}, v_{14}\right\}$. Then, $v_{11} v_{12} \in E$ and $v_{13} v_{14} \in E$. In Observation 2, taking $V^{\prime}=\left(V_{11} \backslash\right.$ $\left.\left\{v_{6}, v_{8}\right\}\right) \cup\{v\}$, we have $n^{\prime}=n-10, m^{\prime}=n-13$ and $\left|T^{\prime}\right| \leq(2 n-23) / 4$. Thus, $T=T^{\prime} \cup\left\{v, v_{4}, v_{5}, v_{9}, v_{11}\right\}$ is a transversal of $H_{G}$ of size at most $(2 n-3) / 4$, contradicting Observation 1 . Hence, $v_{6}$ and $v_{8}$ have a common neighbor, and so $G=F_{11}$.

Suppose secondly that $v_{5}$ and $v_{6}$, or $v_{7}$ and $v_{8}$, have a common neighbor. We may assume that $v_{5}$ and $v_{6}$ have a common neighbor; that is, $N\left(v_{6}\right)=\left\{v_{2}, v_{9}, v_{10}\right\}$. Suppose that $v_{7}$ and $v_{8}$ have no common neighbor. Let $N\left(v_{7}\right)=\left\{v_{3}, v_{11}, v_{12}\right\}$ and $N\left(v_{8}\right)=\left\{v_{4}, v_{13}, v_{14}\right\}$. Then, $v_{11} v_{12} \in E$ and $v_{13} v_{14} \in E$. In Observation 2, taking $V^{\prime}=\left(V_{11} \backslash\left\{v_{7}, v_{8}\right\}\right) \cup\{v\}$, we have $n^{\prime}=n-10, m^{\prime}=n-13$ and $\left|T^{\prime}\right| \leq(2 n-23) / 4$. Thus, $T=T^{\prime} \cup\left\{v, v_{4}, v_{5}, v_{9}, v_{11}\right\}$ is a transversal of $H_{G}$ of size at most $(2 n-3) / 4$, contradicting Observation 1 . Hence, $v_{7}$ and $v_{8}$ have a common neighbor, and so $G=F_{12}$.

Hence we may assume that no two vertices in $\left\{v_{5}, v_{6}, v_{7}, v_{8}\right\}$ have a common neighbor, for otherwise $G \in\left\{F_{11}, F_{12}\right\}$, as desired. Let $N\left(v_{6}\right)=\left\{v_{2}, v_{11}, v_{12}\right\}, N\left(v_{7}\right)=\left\{v_{3}, v_{13}, v_{14}\right\}$, and $N\left(v_{8}\right)=\left\{v_{4}, v_{15}, v_{16}\right\}$. Then, $\left\{v_{9} v_{10}, v_{11} v_{12}, v_{13} v_{14}, v_{15} v_{16}\right\} \subset E$.

Suppose there is an edge joining two triangles each of which contain a vertex from $\left\{v_{5}, v_{6}, v_{7}, v_{8}\right\}$. We may assume that $v_{10} v_{11} \in E$. In Observation 2, taking $V^{\prime}=\left(V_{6} \backslash\right.$ $\left.\left\{v_{4}\right\}\right) \cup\left\{v, v_{10}, v_{11}\right\}$, we have $n^{\prime}=n-8, m^{\prime}=n-12$ and $\left|T^{\prime}\right| \leq(2 n-20) / 4$. Thus, $T=T^{\prime} \cup\left\{v, v_{3}, v_{10}, v_{11}\right\}$ is a transversal of $H_{G}$ of size at most $(2 n-4) / 4$, contradicting Observation 1. Hence there is no edge joining two triangles each of which contain a vertex from $\left\{v_{5}, v_{6}, v_{7}, v_{8}\right\}$.

Suppose there is a vertex, $v_{17}$ say, that is adjacent to two vertices that belong to distinct triangles each of which contain a vertex from $\left\{v_{5}, v_{6}, v_{7}, v_{8}\right\}$. Up to symmetry, there are two cases to consider. Suppose, first, that the vertex $v_{17}$ satisfies $N\left(v_{17}\right)=$ $\left\{v_{9}, v_{10}, v_{11}\right\}$. In Observation 2, taking $V^{\prime}=\left\{v, v_{1}, v_{2}, v_{5}, v_{6}, v_{9}, v_{10}, v_{11}, v_{17}\right\}$, we have $n^{\prime}=n-9, m^{\prime}=n-12$ and $\left|T^{\prime}\right| \leq(2 n-21) / 4$. Thus, $T=T^{\prime} \cup\left\{v, v_{1}, v_{11}, v_{17}\right\}$ is a transversal of $H_{G}$ of size at most $(2 n-5) / 4$, contradicting Observation 1. Suppose, second, that that the vertex $v_{17}$ satisfies $N\left(v_{17}\right)=\left\{v_{9}, v_{10}, v_{13}\right\}$. In Observation 2, taking $V^{\prime}=\left\{v, v_{1}, v_{3}, v_{5}, v_{7}, v_{9}, v_{10}, v_{13}, v_{17}\right\}$, we have $n^{\prime}=n-9, m^{\prime}=n-12$ and $\left|T^{\prime}\right| \leq$ $(2 n-21) / 4$. Thus, $T=T^{\prime} \cup\left\{v, v_{1}, v_{13}, v_{17}\right\}$ is a transversal of $H_{G}$ of size at most $(2 n-5) / 4$, contradicting Observation 1.

Hence there is no vertex that is adjacent to two vertices that belong to distinct triangles each of which contain a vertex from $\left\{v_{5}, v_{6}, v_{7}, v_{8}\right\}$. Thus in Observation 2, taking $V^{\prime}=$ $V_{4} \cup\left\{v_{9}, v_{11}, v_{13}\right\}$, we have $n^{\prime}=n-8, m^{\prime} \leq n-15$ and $\left|T^{\prime}\right| \leq(2 n-23) / 4$. Thus, 
$T=T^{\prime} \cup\left\{v, v_{4}, v_{9}, v_{11}, v_{13}\right\}$ is a transversal of $H_{G}$ of size at most $(2 n-3) / 4$, contradicting Observation 1. This completes the proof of Observation 16.

By Observation 16, we may assume that at least one neighbor of $v$ has degree 4 . We may assume $d\left(v_{1}\right)=4$. Let $N\left(v_{1}\right)=\left\{v, v_{2}, v_{5}, v_{9}\right\}$. Then, $v_{5} v_{9}$ is an edge. By Observation 15, $v_{9}$ is adjacent to no vertex in $\left\{v_{6}, v_{7}, v_{8}\right\}$.

Observation 17 For $i \in\{1,2,3,4\}$, if $d\left(v_{i}\right)=4$, then the two neighbors of $v_{i}$ in $V \backslash N[v]$ have no common neighbor other than $v_{i}$.

Proof. For notational convenience, consider the vertex $v_{1}$. Suppose that $v_{5}$ and $v_{9}$ have a common neighbor different from $v_{1}$. Then, by Observation $12, d\left(v_{5}\right)=d\left(v_{9}\right)=3$. By Observation 15, we may assume that such a common neighbor of $v_{5}$ and $v_{9}$ is adjacent to no vertex in $\left\{v_{2}, v_{3}, v_{4}\right\}$. Let $v_{10}$ be the common neighbor of $v_{5}$ and $v_{9}$ different from $v_{1}$. Since $d\left(v, v_{10}\right)=3, d\left(v_{10}\right)=3$ by Observation 4 .

If $v_{6} v_{10}$ is an edge, then in Observation 2, taking $V^{\prime}=V_{2} \cup\left\{v, v_{4}, v_{5}, v_{6}, v_{9}, v_{10}\right\}$, we have $n^{\prime}=n-8, m^{\prime} \leq n-11$ and $\left|T^{\prime}\right| \leq(2 n-19) / 4$. Thus, $T=T^{\prime} \cup\left\{v, v_{4}, v_{6}, v_{10}\right\}$ is a transversal of $H_{G}$ of size at most $(2 n-3) / 4$, contradicting Observation 1 . Hence, $v_{6} v_{10}$ is not an edge. If $v_{10}$ is adjacent to $v_{7}$ or to $v_{8}$, say $v_{7}$, then in Observation 2, taking $V^{\prime}=V_{3} \cup\left\{v, v_{5}, v_{7}, v_{9}, v_{10}\right\}$, we have $n^{\prime}=n-8, m^{\prime} \leq n-11$ and $\left|T^{\prime}\right| \leq(2 n-19) / 4$. Thus, $T=T^{\prime} \cup\left\{v, v_{2}, v_{7}, v_{10}\right\}$ is a transversal of $H_{G}$ of size at most $(2 n-3) / 4$, contradicting Observation 1. Hence, $v_{10}$ is not adjacent to any vertex in $\left\{v_{6}, v_{7}, v_{8}\right\}$. Let $N\left(v_{10}\right)=$ $\left\{v_{5}, v_{9}, v_{11}\right\}$.

If $v_{6} v_{11}$ is an edge, then in Observation 2, taking $V^{\prime}=V_{2} \cup\left\{v, v_{5}, v_{6}, v_{9}, v_{10}, v_{11}\right\}$, we have $n^{\prime}=n-8, m^{\prime} \leq n-11$ and $\left|T^{\prime}\right| \leq(2 n-19) / 4$. Thus, $T=T^{\prime} \cup\left\{v, v_{1}, v_{6}, v_{11}\right\}$ is a transversal of $H_{G}$ of size at most $(2 n-3) / 4$, contradicting Observation 1 . Hence, $v_{6} v_{11}$ is not an edge. If $v_{11}$ is adjacent to $v_{7}$ or to $v_{8}$, say $v_{7}$, then in Observation 2, taking $V^{\prime}=\left\{v, v_{1}, v_{3}, v_{5}, v_{7}, v_{9}, v_{10}, v_{11}\right\}$, we have $n^{\prime}=n-8, m^{\prime} \leq n-11$ and $\left|T^{\prime}\right| \leq(2 n-19) / 4$. Thus, $T=T^{\prime} \cup\left\{v, v_{1}, v_{7}, v_{11}\right\}$ is a transversal of $H_{G}$ of size at most $(2 n-3) / 4$, contradicting Observation 1 . Hence, $v_{11}$ is not adjacent to any vertex in $\left\{v_{6}, v_{7}, v_{8}\right\}$.

By Observation $4, d\left(v_{11}\right)=3$. Let $N\left(v_{11}\right)=\left\{v_{10}, v_{12}, v_{13}\right\}$. Then, $v_{12} v_{13}$ is an edge. By Observation $4, d\left(v_{12}\right)=d\left(v_{13}\right)=3$. In Observation 2, take $V^{\prime}=\left\{v, v_{1}, v_{5}, v_{9}, v_{10}, v_{11}, v_{12}\right\}$, so we have $n^{\prime}=n-7, m^{\prime} \leq n-12$ and $\left|T^{\prime}\right| \leq(2 n-19) / 4$. Thus, $T=T^{\prime} \cup\left\{v, v_{1}, v_{11}, v_{12}\right\}$ is a transversal of $H_{G}$ of size at most $(2 n-3) / 4$, contradicting Observation 1.

By Observation 17, the vertex $v_{1}$ is the only common neighbor of $v_{5}$ and $v_{9}$. Every neighbor of $v_{3}$ or $v_{4}$ different from $v$ is at distance 3 from $v_{1}$ and therefore has degree 3 by Observation 4 . In particular, $d\left(v_{7}\right)=d\left(v_{8}\right)=3$.

Observation $18 d\left(v_{3}\right)=4$ or $d\left(v_{4}\right)=4$.

Proof. Suppose that $d\left(v_{3}\right)=d\left(v_{4}\right)=3$. Let $N\left(v_{7}\right)=\left\{v_{3}, v_{10}, v_{11}\right\}$. Suppose $v_{7}$ and $v_{8}$ have a common neighbor. Then, $N\left(v_{8}\right)=\left\{v_{4}, v_{10}, v_{11}\right\}$. In Observation 2, taking $V^{\prime}=\left\{v, v_{1}, v_{3}, v_{4}, v_{7}, v_{8}, v_{10}, v_{11}\right\}$, we have $n^{\prime}=n-8, m^{\prime} \leq n-11$ and $\left|T^{\prime}\right| \leq(2 n-19) / 4$. Thus, $T=T^{\prime} \cup\left\{v, v_{1}, v_{7}, v_{10}\right\}$ is a transversal of $H_{G}$ of size at most $(2 n-3) / 4$, contradicting 
Observation 1. Hence, $v_{7}$ and $v_{8}$ have no common neighbor. Let $N\left(v_{8}\right)=\left\{v_{4}, v_{12}, v_{13}\right\}$. Then, $v_{10} v_{11}$ is an edge and $v_{12} v_{13}$ is an edge.

Suppose there is an edge joining a vertex in $\left\{v_{10}, v_{11}\right\}$ and a vertex in $\left\{v_{12}, v_{13}\right\}$. We may assume $v_{11} v_{12}$ is an edge. In Observation 2, taking $V^{\prime}=\left\{v, v_{1}, v_{3}, v_{4}, v_{7}, v_{8}, v_{11}, v_{12}\right\}$, we have $n^{\prime}=n-8, m^{\prime} \leq n-13$ and $\left|T^{\prime}\right| \leq(2 n-21) / 4$. Thus, $T=T^{\prime} \cup\left\{v, v_{1}, v_{11}, v_{12}\right\}$ is a transversal of $H_{G}$ of size at most $(2 n-5) / 4$, contradicting Observation 1 . Hence there is no edge joining a vertex in $\left\{v_{10}, v_{11}\right\}$ and a vertex in $\left\{v_{12}, v_{13}\right\}$.

Suppose that a vertex in $\left\{v_{10}, v_{11}\right\}$ and a vertex in $\left\{v_{12}, v_{13}\right\}$ have a common neighbor, say $v_{13}$. We may assume that $N\left(v_{13}\right)=\left\{v_{10}, v_{11}, v_{12}\right\}$. Then in Observation 2, taking $V^{\prime}=\left\{v, v_{3}, v_{7}, v_{10}, v_{11}, v_{13}\right\}$, we have $n^{\prime}=n-7, m^{\prime} \leq n-12$ and $\left|T^{\prime}\right| \leq(2 n-19) / 4$. Thus, $T=T^{\prime} \cup\left\{v, v_{1}, v_{11}, v_{13}\right\}$ is a transversal of $H_{G}$ of size at most $(2 n-5) / 4$, contradicting Observation 1 . Hence a vertex in $\left\{v_{10}, v_{11}\right\}$ and a vertex in $\left\{v_{12}, v_{13}\right\}$ have no common neighbor.

Suppose that there are two edges joining $\left\{v_{5}, v_{9}\right\}$ and $\left\{v_{10}, v_{11}\right\}$. We may assume that $v_{5} v_{10}$ and $v_{9} v_{11}$ are edges. Then in Observation 2 , taking $V^{\prime}=\left\{v, v_{1}, v_{2}, v_{3}, v_{5}, v_{7}, v_{9}, v_{10}\right.$, $\left.v_{11}\right\}$, we have $n^{\prime}=n-9, m^{\prime} \leq n-11$ and $\left|T^{\prime}\right| \leq(2 n-20) / 4$. Thus, $T=T^{\prime} \cup\left\{v, v_{2}, v_{5}, v_{10}\right\}$ is a transversal of $H_{G}$ of size at most $(2 n-4) / 4$, contradicting Observation 1 . Hence there is at most one edge joining $\left\{v_{5}, v_{9}\right\}$ and $\left\{v_{10}, v_{11}\right\}$. Similarly, there is at most one edge joining $\left\{v_{5}, v_{9}\right\}$ and $\left\{v_{12}, v_{13}\right\}$. We may therefore assume that there is no edge joining $\left\{v_{5}, v_{9}\right\}$ and $\left\{v_{10}, v_{12}\right\}$. Hence in Observation 2, taking $V^{\prime}=\left\{v, v_{1}, v_{3}, v_{4}, v_{10}, v_{12}\right\}$, we have $n^{\prime}=n-6, m^{\prime} \leq n-13$ and $\left|T^{\prime}\right| \leq(2 n-19) / 4$. Thus, $T=T^{\prime} \cup\left\{v, v_{1}, v_{10}, v_{12}\right\}$ is a transversal of $H_{G}$ of size at most $(2 n-4) / 4$, contradicting Observation 1.

By Observation 18, we may assume that $v_{3}$ or $v_{4}$, say $v_{4}$, has degree 4 . Let $N\left(v_{4}\right)=$ $\left\{v, v_{3}, v_{8}, v_{10}\right\}$. Then, $v_{8} v_{10}$ is an edge. Every vertex at distance 2 from $v$ is at distance 3 from either $v_{1}$ or $v_{4}$ and therefore, by Observation 4, has degree 3. By Observation 17, $v_{4}$ is the only common neighbor of $v_{8}$ and $v_{10}$. By the claw-freeness of $G$, and by Observations 15 and 17, no two vertices at distance 2 from $v$ have a common neighbor in $V \backslash N(v)$.

Observation $19 d\left(v_{3}\right)=3$.

Proof. Suppose that $d\left(v_{3}\right)=4$. Let $N\left(v_{3}\right)=\left\{v, v_{4}, v_{7}, v_{11}\right\}$. Then, $v_{7} v_{11}$ is an edge. For $i \in\{7,8,10,11\}$, let $v_{i}^{\prime}$ be the neighbor of $v_{i}$ at distance 3 from $v$. Hence, $N\left(v_{7}\right)=\left\{v_{3}, v_{7}^{\prime}, v_{11}\right\}$ and $N\left(v_{11}\right)=\left\{v_{3}, v_{7}, v_{11}^{\prime}\right\}$, while $N\left(v_{8}\right)=\left\{v_{4}, v_{8}^{\prime}, v_{10}\right\}$ and $N\left(v_{10}\right)=$ $\left\{v_{4}, v_{8}, v_{10}^{\prime}\right\}$. Let $W=\left\{v_{7}^{\prime}, v_{8}^{\prime}, v_{10}^{\prime}, v_{11}^{\prime}\right\}$.

We show that $W$ is an independent set. Suppose that two vertices in $W$ are adjacent. We may assume that $v_{7}^{\prime} v_{11}^{\prime}$ is an edge or $v_{7}^{\prime} v_{8}^{\prime}$ is an edge. Suppose $v_{7}^{\prime} v_{11}^{\prime}$ is an edge. Then in Observation 2, taking $V^{\prime}=\left\{v, v_{1}, v_{3}, v_{7}, v_{7}^{\prime}, v_{11}, v_{11}^{\prime}\right\}$, we have $n^{\prime}=n-7, m^{\prime} \leq n-12$ and $\left|T^{\prime}\right| \leq(2 n-19) / 4$. Thus, $T=T^{\prime} \cup\left\{v, v_{1}, v_{7}, v_{7}^{\prime}\right\}$ is a transversal of $H_{G}$ of size at most $(2 n-$ 3)/4, contradicting Observation 1. Suppose $v_{7}^{\prime} v_{8}^{\prime}$ is an edge. Let $w$ be the common neighbor of $v_{7}^{\prime}$ and $v_{8}^{\prime}$. Then in Observation 2, taking $V^{\prime}=\left\{v, v_{3}, v_{4}, v_{5}, v_{6}, v_{7}, v_{7}^{\prime}, v_{8}, v_{8}^{\prime}, w\right\}$, we have $n^{\prime}=n-10, m^{\prime} \leq n-17$ and $\left|T^{\prime}\right| \leq(2 n-27) / 4$. Thus, $T=T^{\prime} \cup\left\{v_{3}, v_{4}, v_{5}, v_{6}, v_{7}^{\prime}, w\right\}$ is a transversal of $H_{G}$ of size at most $(2 n-3) / 4$, contradicting Observation 1 . Thus, $W$ is an independent set. 
If no two vertices in $W$ have a common neighbor, then in Observation 2, taking $V^{\prime}=\left\{v, v_{1}, v_{3}, v_{4}\right\} \cup W$, we have $n^{\prime}=n-8, m^{\prime} \leq n-19$ and $\left|T^{\prime}\right| \leq(2 n-27) / 4$. Thus, $T=T^{\prime} \cup\left\{v, v_{1}\right\} \cup W$ is a transversal of $H_{G}$ of size at most $(2 n-3) / 4$, contradicting Observation 1. Hence, two vertices in $W$ have a common neighbor.

Suppose $v_{7}^{\prime}$ and $v_{11}^{\prime}$ or $v_{8}^{\prime}$ and $v_{10}^{\prime}$, say $v_{7}^{\prime}$ and $v_{11}^{\prime}$, have a common neighbor. Let $N\left(v_{7}^{\prime}\right)=\left\{v_{7}, v_{12}, v_{13}\right\}$. Then, $N\left(v_{11}^{\prime}\right)=\left\{v_{11}, v_{12}, v_{13}\right\}$. In Observation 2, taking $V^{\prime}=$ $\left\{v, v_{3}, v_{7}, v_{7}^{\prime}, v_{11}, v_{11}^{\prime}, v_{12}, v_{13}\right\}$, we have $n^{\prime}=n-8, m^{\prime} \leq n-11$ and $\left|T^{\prime}\right| \leq(2 n-19) / 4$. Thus, $T=T^{\prime} \cup\left\{v, v_{3}, v_{7}^{\prime}, v_{12}\right\}$ is a transversal of $H_{G}$ of size at most $(2 n-3) / 4$, contradicting Observation 1. Hence, neither $v_{7}^{\prime}$ and $v_{11}^{\prime}$ nor $v_{8}^{\prime}$ and $v_{10}^{\prime}$ have a common neighbor. Hence a vertex in $\left\{v_{7}^{\prime}, v_{11}^{\prime}\right\}$ and a vertex in $\left\{v_{8}^{\prime}, v_{10}^{\prime}\right\}$ have a common neighbor. We may assume that $v_{7}^{\prime}$ and $v_{8}^{\prime}$ have a common neighbor. Let $N\left(v_{7}^{\prime}\right)=\left\{v_{7}, v_{12} v_{13}\right\}$. Then, $N\left(v_{8}^{\prime}\right)=$ $\left\{v_{8}, v_{12}, v_{13}\right\}$. In Observation 2, taking $V^{\prime}=\left\{v_{3}, v_{4}, v_{7}, v_{7}^{\prime}, v_{8}, v_{8}^{\prime}, v_{12}, v_{13}\right\}$, we have $n^{\prime}=$ $n-8, m^{\prime} \leq n-11$ and $\left|T^{\prime}\right| \leq(2 n-19) / 4$. Thus, $T=T^{\prime} \cup\left\{v_{3}, v_{4}, v_{7}^{\prime}, v_{12}\right\}$ is a transversal of $H_{G}$ of size at most $(2 n-3) / 4$, contradicting Observation 1 .

By Observation 19, $d\left(v_{3}\right)=3$. An identical argument (interchanging the roles of $v_{3}$ and $v_{4}$ with $v_{1}$ and $v_{2}$ ) shows that $d\left(v_{2}\right)=3$. Let $N\left(v_{6}\right)=\left\{v_{2}, v_{12}, v_{13}\right\}$ and $N\left(v_{7}\right)=$ $\left\{v_{3}, v_{14}, v_{15}\right\}$. Then, $v_{12} v_{13}$ is an edge and $v_{14} v_{15}$ is an edge. An identical proof as the proof of Observation 18 now produces a contradiction. This completes the proof of Theorem 9.

\section{References}

[1] D. Archdeacon, J. Ellis-Monaghan, D. Fischer, D. Froncek, P.C.B. Lam, S. Seager, B. Wei, and R. Yuster, Some remarks on domination. J. Graph Theory 46 (2004), 207-210.

[2] V. Chvátal and C. McDiarmid, Small transversals in hypergraphs. Combinatorica 12 (1992), 19-26.

[3] E. J. Cockayne, R. M. Dawes, and S. T. Hedetniemi, Total domination in graphs. Networks 10 (1980), 211-219.

[4] O. Favaron and M. A. Henning, Paired domination in claw-free cubic graphs. Graphs Combin. 20 (2004), 447-456.

[5] T. W. Haynes, S. T. Hedetniemi, and P. J. Slater (eds), Fundamentals of Domination in Graphs, Marcel Dekker, Inc. New York, 1998.

[6] T. W. Haynes, S. T. Hedetniemi, and P. J. Slater (eds), Domination in Graphs: Advanced Topics, Marcel Dekker, Inc. New York, 1998.

[7] M. A. Henning, L. Kang, E. Shan, and A Yeo, On matching and total domination in graphs, manuscript (2005). 
[8] M. A. Henning and A. Yeo, Hypergraphs with large transversal number and with edge sizes at least three, manuscript (2005).

[9] M. Las Vergnas, A note on matchings in graphs. Colloque sur la Théorie des Graphes (Paris, 1974), Cahiers Centre Étude Rech. Opér. 17 (1975), 257-260.

[10] M. Plummer, Factors and Factorization. 403-430. Handbook of Graph Theory ed. J. L. Gross and J. Yellen. CRC Press, 2003, ISBN: 1-58488-092-2.

[11] W. R. Pulleyblank, Matchings and Extension. 179-232. Handbook of Combinatorics ed. R. L. Graham, M. Grötschel, L. Lovász. Elsevier Science B.V. 1995, ISBN 0-44482346-8.

[12] D. Sumner, On Tutte's factorization theorem. Graphs and Combinatorics, Lecture Notes in Math. Vol 406, Springer (1974), 350-355.

[13] D. Sumner, 1-factors and anti-factor sets. J. London Math. Soc. 13 (1976), 351-359.

[14] S. Thomassé and A. Yeo, Total domination of graphs and small transversals of hypergraphs. To appear in Combinatorica.

[15] Z. Tuza, Covering all cliques of a graph. Discrete Math. 86 (1990), 117-126. 